\title{
COMERCIANDO CON INCERTIDUMBRE: LOS MERCADOS DE AGUA EN LA AGRICULTURA CHILENA*
}

\author{
ERENEY HADJIGEORGALIS **
}

\begin{abstract}
This research examines the effect of uncertainty on farmers' water trading decisions in the Limari River basin. The results show that farmers who face greater risks from water supply shortfalls, such as perennial crop farmers, will not participate in spot water markets but rather in permanent water rights markets. In addition, transactions will be generated not only by differences in the value of the marginal product of water, but also by differences in irrigation efficiency and for risk sharing motives. The main policy implication is that water rights hoarding may be efficient in a water market when it leads to efficient risk sharing among farmers.
\end{abstract}

\footnotetext{
Se agradece a los Profesores Lowell Jarvis, Richard Howitt y Art Havenner de la Universidad de California, Davis, al Profesor Octavio Ramirez de New Mexico State University, y a un árbitro anónimo por sus comentarios y sugerencias sobre este manuscrito. También se agradece a la Sra. Sylvia Beuhler de New Mexico State University por su excelente traducción a castellano de la versión original en inglés. Todos los errores restantes son de responsabilidad única de la autora. Esta investigación se financió con el apoyo de la Social Science Research Council, con fondos provenientes de la Fundación Ford, del programa Fulbright de USIA y del College of Agriculture y Programa Pacific Rim de la Universidad de California.

** Assistant Professor, New Mexico State University, Department of Agricultural Economics and Agricultural Business. E.mail: ereney@nmsu.edu JEL classification: Q25

Keywords: Water Markets, Risk, Uncertainty, Limarí River Basin.
} 


\section{RESUMEN}

Esta investigación examina el efecto de la incertidumbre sobre las decisiones de compraventa en dos mercados de agua en la Cuenca de Limarí: un mercado spot, donde se transan volúmenes de agua por una temporada y un mercado de derechos de aprovechamiento de agua. Los resultados demuestran que aquellos agricultores que enfrentan mayor riesgo de un déficit de agua, tales como los productores de cultivos permanentes, no participarán en mercados spot sino en mercados de derechos de aprovechamiento de agua. Además las transacciones de agua se generarán tanto por diferencias en eficiencia de riego y tolerancias al riesgo como por diferencias en el valor del producto marginal de agua. La implicancia principal para la política es que el acaparamiento de derechos de aprovechamiento de agua podría ser eficiente en un mercado de agua cuando conduzca a una repartición eficiente de riesgo entre agricultores.

\section{INTRODUCCIÓN}

La última década ha sido testigo de un interés creciente en asignar los recursos hídricos a través de mercados privados (Bauer, 1998; Beare et al., 1998; Howitt, 1998; Rosegrant y Binswanger, 1994; Colby, 1990; Colby-Saliba y Bush, 1987; Anderson, 1983). La reasignación de las dotaciones iniciales de agua se efectúa a través de los precios del mercado, los cuales señalan el valor del agua en sus distintos usos. En la agricultura, se espera que estos precios sean iguales al valor del producto marginal de agua. El valor del producto marginal del agua, sin embargo, es una medida inadecuada del valor del agua en la agricultura dada la incertidumbre asociada con el abastecimiento de agua y la producción agrícola, los distintos riesgos que esta incertidumbre impone en los agricultores, y las distintas preferencias de riesgo que caracterizan a cada agricultor.

La producción agrícola está rodeada de incertidumbre. Entre la siembra y la cosecha pueden ocurrir eventos aleatorios que afectan los ingresos finales del agricultor, tales como plagas, sequías y cambios bruscos en los precios de sus productos. Además, la producción agrícola en muchas zonas de Chile depende fuertemente del regadío, lo cual introduce la incertidumbre adicional del abastecimiento de agua.

La incertidumbre sobre el abastecimiento de agua impone distintos riesgos a los agricultores, dependiendo de cuán sensible es su producción a variabilidad en la disponibilidad de agua. Los productores de cultivos permanentes que han hecho inversiones sustanciales en plantaciones, por ejemplo, enfrentan un riesgo mucho mayor que aquellos agricultores que producen solamente cultivos anuales. Estos agricultores arriesgan pérdidas no solamente en la producción actual, sino también a futuro si el abastecimiento de agua no es suficiente para satisfacer sus necesidades de riego. 
Finalmente, los agricultores difieren en su tolerancia al riesgo. Aunque muchos factores pueden influir en la tolerancia al riesgo de un agricultor, esta investigación se enfoca en la relación entre la tolerancia al riesgo y el ingreso. En la medida que aumenta el ingreso del agricultor, éste será más tolerante al riesgo y estará dispuesto a tomar decisiones más riesgosas en su producción agrícola que le ofrecen un mayor potencial de ganancia. Al contrario, a niveles bajos de ingreso, el agricultor preferirá un ingreso seguro a un ingreso potencial mayor pero incierto.

Las consecuencias que tiene la incertidumbre, los riesgos asociados con ella y la tolerancia al riesgo para las decisiones en un mercado de agua no se han abordado en la literatura hasta la fecha. Esta investigación plantea la hipótesis de que la incorporación del riesgo y de la incertidumbre en la valoración del agua y de los derechos de aprovechamiento de ésta resultará en patrones de comercio distintos de aquellos esperados cuando se toman en cuenta sólo el valor del producto marginal del agua y los costos de transacción. Específicamente, se mostrará cómo el riesgo y la incertidumbre determinan quién participará en los distintos mercados de agua y los patrones de comercio en estos mercados, ocupando datos de transacciones de agua recopilados en la cuenca del Río Limarí a través de una encuesta de más de 300 agricultores durante la temporada 1996/97.

La cuenca del Río Limarí es un excelente estudio de caso para los mercados de agua por varias razones. Primero, tiene una larga trayectoria de comerciar en agua. Aunque los derechos de aprovechamiento de agua en Chile no se privatizaron hasta 1981, las transacciones informales en la cuenca del Río Limarí se realizaron tan tempranamente como en 1940. Además, la cuenca del Río Limarí es la única en Chile que mantiene dos mercados activos de aguas: un mercadospot, donde se transan volúmenes de agua por una temporada, y un mercado de derechos de aprovechamiento de agua.

El artículo se organiza de la siguiente manera. Primero, se expone el modelo conceptual, donde se derivan las hipótesis a probar. Después se presenta la base de datos y se analizan algunas estadísticas sumarias claves del estudio. En seguida se expone el modelo empírico, seguido del análisis de los resultados y finalmente, las conclusiones.

\section{Modelo Conceptual}

Cuando se privatiza el derecho de aprovechamiento de agua, ésta se transforma de un simple insumo en el proceso de producción agrícola a un insumoactivo. En la ausencia de un mercado de agua, el productor decide cuánto terreno dedicar a la producción regada y cuánto dejar en barbecho. Cuando existe un mercado de agua, sin embargo, surgen las opciones adicionales de la compraventa de volúmenes de agua y de los derechos de su aprovechamiento. En este caso, podría ser rentable para los productores de cultivos de bajo valor reducir su producción regada para vender volúmenes de agua en el mercado spot. También se 
podría observar el traspaso de derechos de aprovechamiento de agua de usos de bajo a usos de alto valor.

¿Cómo se toman estas decisiones económicas con respecto a la producción agrícola y a las transacciones de agua? En el fondo, los agricultores se preocupan por maximizar sus beneficios a largo plazo. Pero las decisiones con respecto al agua no se pueden modelar en un sencillo cuadro de maximización de beneficios debido a varias fuentes de incertidumbre. Específicamente, se refiere a la incertidumbre del abastecimiento de agua a futuro, de los precios de producción y de los precios volátiles de volúmenes de agua en el mercado de una temporada a otra. Esta incertidumbre obliga a los agricultores a tomar sus decisiones más bien en base de sus expectativas. Por lo tanto, el objetivo ya no es maximizar los beneficios a largo plazo, sino la utilidad esperada de tales beneficios, lo cual incorpora estos elementos aleatorios.

El modelo completo de la maximización de la utilidad esperada de los beneficios a largo plazo, sujeto a las restricciones de recursos disponibles al agricultor, se encuentra en el Apéndice I y las correspondientes condiciones de primer orden en el Apéndice II. De estas condiciones de primer orden, se pueden derivar los precios de reserva de la compraventa de volúmenes de agua y de los derechos de aprovechamiento del agua (Apéndices III, IV y V). Estos precios de reserva son la base del comercio en el mercado de agua e ilustran las tres fuentes de riesgo enfrentadas por los agricultores cuando toman sus decisiones sobre el abastecimiento de agua. Cada riesgo tiene un costo de incertidumbre asociado con él que variará entre los agricultores. En las siguientes secciones se derivan las hipótesis a probar que conforman la base del modelo empírico. Las definiciones de los símbolos utilizados se encuentran en el Apéndice VI.

2.1. El riesgo y la incertidumbre en los mercados de derechos de aprovechamiento de agua

En los mercados de derechos de aprovechamiento de agua los agricultores enfrentan incertidumbre sobre el abastecimiento futuro de ésta, la cual se refleja en su precio de reserva por un derecho de aprovechamiento de agua:

$$
\overline{\mathrm{P}}_{\mathrm{Rt}}^{\mathrm{DAA}} \approx \sum_{\tau=1}^{\mathrm{T}} \beta_{\mathrm{t}}\left[\overline{\mathrm{P}}_{\mathrm{t}}^{\mathrm{I}} \frac{\partial \mathrm{f}(\bullet)}{\partial \mathrm{e}_{\mathrm{i}} \mathrm{W}_{\mathrm{t}}^{\mathrm{I}}} \mathrm{e}_{\mathrm{i}}-\frac{1}{2} \overline{\mathrm{R}} \frac{\partial \sigma_{\pi}^{2}}{\partial \mathrm{e}_{\mathrm{i}} \mathrm{W}_{\mathrm{t}}^{\mathrm{I}}} \mathrm{e}_{\mathrm{i}}-\mathrm{c}_{\mathrm{t}}^{\mathrm{R}}\right]
$$

donde $\overline{\mathrm{R}}$ es el coeficiente "Arrow-Pratt" de la aversión absoluta al riesgo y $\sigma_{\pi}^{2}$ es la varianza del ingreso. El primer término representa el valor presente de los beneficios del riego medidos por el valor de producto marginal (VPM) esperado del agua ajustado por la eficiencia de riego $\left(\mathrm{e}_{\mathrm{i}}\right)$. El segundo término representa el costo marginal de la incertidumbre asociada con el abastecimiento de agua a futuro. Este costo es equivalente al cambio en la varianza del ingreso causado por un cambio en la cantidad de agua aplicada en riego. 
Si la disponibilidad de agua a un agricultor varía poco, el costo de la incertidumbre será cercano a cero. Para aquellos agricultores cuya disponibilidad de agua es más variable, sin embargo, este costo podría ser significativo, teniendo el efecto de reducir substancialmente el precio de reserva del agricultor en el mercado. Además, mientras mayores sean el producto marginal del agua y la eficiencia de riego, más alto será el precio de reserva de un derecho de aprovechamiento de agua de un agricultor. Los agricultores que invierten en la tecnificación del riego, mediante tranques de acumulación y/o riego por goteo, por lo tanto, tendrán mayor inclinación a ser compradores en un mercado de agua. Esto contradice la creencia común de que las inversiones en tecnologías que incrementan la eficiencia del riego liberarán agua para la venta en el mercado. Por lo tanto, se espera que las transacciones de derechos de aprovechamiento de agua ocurran desde agricultores con abastecimientos de agua menos seguros a aquellos con abastecimientos más seguros, o desde agricultores con baja eficiencia de riego y/o un bajo VPM esperado de agua a agricultores con mayor eficiencia de riego y/o VPM esperado de agua.

\subsection{El riesgo y la incertidumbre en el mercado spot}

Los mercados spot son más riesgosos que los mercados de derechos de aprovechamiento de agua, ya que los agricultores que participan en éstos enfrentan no solamente incertidumbre sobre el abastecimiento de agua a futuro, sino también incertidumbre sobre los precios del mercado mismo de una temporada a otra, lo que afecta sus niveles de ingreso. No obstante, este último riesgo es diferente para los agricultores que venden volúmenes de agua comparado con aquellos que los compran. Esta asimetría entre compradores y vendedores se observa en sus precios de reserva de compra y venta de volúmenes de agua en el mercado spot:

$$
\begin{aligned}
& \overline{\mathrm{P}}_{\mathrm{Rt}}^{\mathrm{SC}} \approx \mathrm{e}_{\mathrm{i}}\left[\overline{\mathrm{P}}_{\mathrm{t}}^{\mathrm{I}} \frac{\partial \mathrm{f}(\bullet)}{\partial \mathrm{W}_{\mathrm{t}}^{\mathrm{I}}}\right]-\frac{1}{2} \overline{\mathrm{R}}\left[\frac{\partial \sigma_{\pi}^{2}}{\partial \mathrm{e}_{\mathrm{i}} \mathrm{W}_{\mathrm{t}}^{\mathrm{I}}} \mathrm{e}_{\mathrm{i}}+\frac{\partial \sigma_{\pi}^{2}}{\partial \mathrm{W}_{\mathrm{t}}^{\mathrm{B}}}\right] \\
& \overline{\mathrm{P}}_{\mathrm{Rt}}^{\mathrm{SV}} \approx \mathrm{e}_{\mathrm{i}}\left[\overline{\mathrm{P}}_{\mathrm{t}}^{\mathrm{I}} \frac{\partial \mathrm{f}(\bullet)}{\partial \mathrm{e}_{\mathrm{i}} \mathrm{W}_{\mathrm{t}}^{\mathrm{I}}}\right]-\frac{1}{2} \overline{\mathrm{R}}\left[\frac{\partial \sigma_{\pi}^{2}}{\partial \mathrm{e}_{\mathrm{i}} \mathrm{W}_{\mathrm{t}}^{\mathrm{I}}} \mathrm{e}_{\mathrm{i}}-\frac{\partial \sigma_{\pi}^{2}}{\partial \mathrm{W}_{\mathrm{t}}^{\mathrm{S}}}\right]
\end{aligned}
$$

El primer término en el precio de reserva de ambos participantes es igual: el VPM esperado del agua ajustado por la eficiencia de riego. Los segundos términos representan los costos asociados con la incertidumbre para los compradores y los vendedores, los cuales combinan el costo de la incertidumbre sobre el abastecimiento futuro de agua $\left(\partial \sigma_{\pi}^{2} / \partial \mathrm{e}_{\mathrm{i}} \mathrm{W}_{\mathrm{t}}^{\mathrm{t}} \mathrm{e}_{\mathrm{i}}\right)$ con la incertidumbre del mercado spot $\left(\partial \sigma_{\pi}^{2} / \partial \mathrm{W}_{\mathrm{t}}^{\mathrm{B}}\right.$ y $\left.\partial \sigma_{\pi}^{2} / \partial \mathrm{W}_{\mathrm{t}}^{\mathrm{S}}\right)$. Para los compradores, el costo de incertidumbre del mer- 
cado spot es equivalente al cambio en la varianza del ingreso causado por un cambio en el volumen de agua comprado $\left(\partial \sigma_{\pi}^{2} / \partial \mathrm{W}_{\mathrm{t}}^{\mathrm{B}}\right)$, mientras que para los vendedores es el cambio en la varianza del ingreso causado por un cambio en el volumen de agua vendido $\left(\partial \sigma_{\pi}^{2} / \partial \mathrm{W}_{\mathrm{t}}^{\mathrm{s}}\right)$.

Sólo los compradores enfrentan incertidumbre sobre el aumento de los precios de agua, conocido como incertidumbre downside, o incertidumbre a pérdidas, en el mercado spot. En años de escasez de agua, los precios en el mercado spot serán altos y, por lo tanto, los agricultores que compran agua experimentarán una reducción en sus beneficios netos. Los vendedores en el mercado spot no corren el mismo riesgo, ya que venderán agua solamente cuando los precios son suficientemente altos para inducirlos a reducir su producción regada. Es decir, cuando las ganancias de la venta de agua son mayores que las de la producción agrícola. Por lo tanto, los vendedores reciben el beneficio de una reducción del riesgo porque el mercado spot les ofrece un ingreso alternativo en años donde la producción regada para ellos no es rentable.

La asimetría de riesgo que enfrentan los compradores y vendedores en el mercado spot tiene una implicancia para los patrones de comercio. Aun cuando el VPM esperado de agua y la eficiencia de riego sean iguales entre agricultores, todavía se podrían observar traspasos de agua entre agricultores para lograr una asignación o repartición eficiente del riesgo .

2.3. El riesgo adicional: el caso especial de los productores de cultivos permanentes

Los productores de cultivos permanentes son un caso especial entre los agricultores en un mercado de agua porque enfrentan el riesgo adicional de la pérdida de su inversión en su stock de cultivos permanentes en caso de un déficit de agua. Este riesgo se incorpora en el valor marginal presente del stock de cultivos permanentes:

$$
\rho_{\text {it }}=\sum_{\tau=1}^{\mathrm{T}} \beta_{\mathrm{t}} \mathrm{V}^{\prime}(\bar{\pi})\left[\begin{array}{llllll}
\eta^{\mathrm{t}-1} & \overline{\mathrm{P}}_{\mathrm{t}}^{\mathrm{I}} & \frac{\partial \mathrm{f}(\bullet)}{\partial \mathrm{K}_{\mathrm{t}}^{\mathrm{p}}} & -\frac{1}{2} & \overline{\mathrm{R}} & \frac{\partial \sigma_{\pi}^{2}}{\partial \mathrm{K}_{\mathrm{t}}^{\mathrm{p}}}
\end{array}\right]+\eta^{\mathrm{T}-1} \rho_{\mathrm{T}}
$$

donde el primer término entre los paréntesis es el VPM esperado del stock de los cultivos permanentes, ajustado por reducciones en productividad a lo largo del tiempo, y el segundo término representa el costo de la incertidumbre asociada con la pérdida potencial del stock de cultivos permanentes de un abastecimiento reducido de agua. El término final afuera del paréntesis es el valor terminal del stock.

El riesgo adicional asociado con los cultivos permanentes tiene dos implicancias importantes para la actividad en el mercado de agua. Primero, los productores de cultivos permanentes no tenderán a participar en el mercadospot. Segundo, ellos tenderán a demandar más derechos de aprovechamiento de agua que los productores de cultivos anuales, lo que hace más probable que participen en el mercado de derechos de aprovechamiento de agua que los productores de cultivos anuales. 
Se espera que los agricultores de cultivos permanentes no tenderán a participar en el mercado spot por dos razones. Primero, tenderán a poner los precios de reserva más altos. Por lo tanto, demandarán un preciospot más alto para entrar al mercado, pero no podrán competir con los agricultores de cultivos anuales y de praderas que están dispuestos a vender agua a precios menores. Por lo tanto, los productores de cultivos permanentes no tenderán a participar como vendedores en el mercado spot.

Segundo, los productores de cultivos permanentes intentarán compensarse por el riesgo adicional que enfrentan a reducir el riesgo que enfrentan en otros sitios del mercado. Por lo tanto, no dependerán del mercado spot para satisfacer sus necesidades de riego, sino que demandarán más derechos de aprovechamiento de agua para garantizar una cantidad mínima de agua en los años secos y para evitar enfrentar el riesgo de precios en el mercado spot. Por ende, no participarán tampoco como compradores en el mercado spot.

\subsection{La aversión al riesgo y la decisión de plantar vs. vender}

El nivel de aversión al riesgo de un agricultor individual influye en sus precios de reserva y, por lo tanto, en su decisión de participar en el mercado del agua. Se dice que los agricultores muestran una aversión absoluta al riesgo decreciente si al aumentar su ingreso están dispuestos a enfrentar más riesgo. A bajos niveles de ingreso, un agricultor preferirá un ingreso bajo pero seguro a un ingreso más alto pero incierto. Es decir, preferirá vender agua en el mercado spot, o derechos de aprovechamiento de agua, en lugar de correr el riesgo asociado con la producción agrícola. Por esta razón, se espera que los agricultores de bajos ingresos y de liquidez limitada venderán agua en el mercado spot o venderán sus derechos de aprovechamiento de agua a aquellos agricultores de mayores ingresos. De esta manera, ocurrirán traspasos de agua con el objetivo de compartir riesgo.

\section{Datos y Estadísticas Sumarias}

\subsection{Datos}

Durante la temporada de 1996/97 se encuestó a una muestra aleatoria estratificada de agricultores con predios ubicados en la cuenca del Río Limarí, tanto bajo como sobre el sistema de distribución de aguas conocido como el Sistema Paloma. Las fuentes de información que se utilizaron en la selección de la muestra incluyeron los registros de derechos de aprovechamiento de agua en el Conservador de Bienes Raíces de Ovalle y los archivos mantenidos por las asociaciones de canalistas y las juntas de vigilancia. Estas fuentes se utilizaron para identificar a aquellos agricultores que habían participado en el mercado de aguas. Se seleccionaron agricultores que no participaron en el mercado por estrato geográfico. 
La distribución geográfica de la muestra incluye 207 agricultores bajo el embalse Paloma del sistema y 109 agricultores sobre el embalse para asegurar una representación de los agricultores en la misma proporción que la superficie total bajo riego en cada zona. Aproximadamente un 67 por ciento de la superficie bajo riego en el valle se ubica bajo el embalse Paloma.

Los datos recopilados en la encuesta incluyen información sobre las características de los predios, los patrones de plantación de cultivos, los precios de producción, las transacciones en el mercado de agua y las características socioeconómicas de los encuestados. La información sobre las transacciones en el mercado de agua incluye todas las transacciones de derechos de aprovechamiento de agua desde 1981 y las transacciones de agua en el mercadospot durante las temporadas de 1995/96 y 1996/97.

\subsection{Estadísticas sumarias}

El Cuadro 1 presenta las estadísticas descriptivas de toda la muestra y de tres subgrupos de agricultores: (1) aquellos con predios ubicados bajo el embalse Paloma (que es la área que define el mercadospot), (2) aquellos que participaron en el mercado spot como compradores y/o vendedores en la temporada 1996/97 y (3) aquellos que habían participado en el mercado de derechos de aprovechamiento de agua como compradores y/o vendedores desde 1981. Las definiciones de las variables se presentan en el Cuadro 2.

Al analizar las estadísticas sumarias, se observan algunas diferencias entre el tipo de agricultor que participa en el mercado de derechos de aprovechamiento de agua y aquel que participa en el mercado spot. Primero, la gran mayoría de los agricultores que han participado en el mercado de derechos de aprovechamiento de agua tienen predios ubicados bajo el embalse Paloma. Solamente 21 por ciento de los agricultores ubicados sobre el embalse Paloma han comprado o vendido derechos de aprovechamiento de agua desde 1981. Por lo tanto, la representación de agricultores con predios ubicados bajo el embalse Paloma en el mercado de derechos de aprovechamiento de agua $(79.3 \%)$ es más que proporcional a la cantidad de superficie regada en esta zona $(67 \%)$.

Segundo, el porcentaje de productores de cultivos permanentes que participaron en el mercado de derechos de aprovechamiento de agua (66.7\%) es significativamente mayor que el porcentaje que participaron en el mercado spot (49.3\%). A primera vista esto apoya la hipótesis de que dichos agricultores tienden a no participar en el mercadospot, sino más bien en el mercado de derechos de aprovechamiento de agua. Además, se observa que los productores de cultivos permanentes que participan en el mercado de derechos de aprovechamiento dedicaron tres veces más superficie a uva de mesa de exportación que los productores de cultivos permanentes que participan en el mercado spot. Esto señala el riesgo adicional de pérdida de stock de cultivos permanentes que enfrentan los agricultores que participan en el mercado de derechos de aprovechamiento de agua. 
CUADRO 1

ESTADISTICAS DESCRIPTIVAS: LOS MEDIOS DE LAS VARIABLES Y LOS PORCENTAJES DE AGRICULTORES POR CATEGORIA*

\begin{tabular}{|c|c|c|c|c|}
\hline Variable & $\begin{array}{c}\text { Todos los } \\
\text { agricultores } \\
\%\end{array}$ & $\begin{array}{c}\text { Los agricultores } \\
\text { bajo el Embalse } \\
\text { Paloma } \\
\%\end{array}$ & $\begin{array}{c}\text { Los agricultores } \\
\text { en el mercado } \\
\text { spot } \\
\%\end{array}$ & $\begin{array}{l}\text { Los agricultores } \\
\text { en el mercado de } \\
\text { derechos de } \\
\text { aprovechamiento } \\
\text { de agua } \%\end{array}$ \\
\hline \multicolumn{5}{|l|}{ Variables binarias } \\
\hline BAJO-EMB & 65,9 & 100,0 & 100,0 & 79,3 \\
\hline REFAG & 41,4 & 35,6 & 33,3 & 43,0 \\
\hline REFAGP & 32,3 & 29,0 & 25,4 & 34,5 \\
\hline COMPRA-D & 9,8 & 13,5 & 16,9 & 36,9 \\
\hline DFAM & 19,9 & 24,6 & 23,9 & 26,4 \\
\hline DPEQ & 44,6 & 35,7 & 33,8 & 33,3 \\
\hline DNOAG & 20,6 & 25,6 & 19,7 & 20,7 \\
\hline DPERM & 68,0 & 54,1 & 49,3 & 66,7 \\
\hline VENDIO-A & 13,6 & 16,4 & 53,5 & 13,8 \\
\hline MULTSEC & 5,1 & 7,2 & 15,5 & 11,5 \\
\hline PARMDA & 27,5 & 33,3 & 31,0 & 100,0 \\
\hline PARMS & 22,5 & 34,3 & 100,0 & 25,3 \\
\hline TANQUE & 38,4 & 42,2 & 50,7 & 36,8 \\
\hline COMPRA-A & 10,4 & 15,9 & 46,5 & 9,2 \\
\hline VENDIO-D & 16,8 & 19,3 & 14,1 & 63,1 \\
\hline TECRIEGO & 26,6 & 26,6 & 28,2 & 33,3 \\
\hline \multicolumn{5}{|l|}{ Variables continuas } \\
\hline HACULT (ha.) & 24,60 & 32,65 & 37,67 & 34,20 \\
\hline HAUVAEX & 0,10 & 0,06 & 0,05 & 0,17 \\
\hline NPREDIOS & 1,38 & 1,49 & 1,676 & 1,655 \\
\hline PORCPERM & 0,36 & 0,26 & 0,25 & 0,39 \\
\hline ANIMTOT & 20,76 & 25,26 & 19,99 & 19,12 \\
\hline CAPALM(1000 m3) & 2,08 & 2,78 & 4,35 & 3,51 \\
\hline EXP & 29,73 & 27,22 & 25,76 & 29,74 \\
\hline
\end{tabular}

Nota:

* Los porcentajes de las variables binarias representan el porcentaje de los agricultores para quienes el valor de la variable es igual a uno. 


\section{CUADRO 2}

\section{LAS DEFINICIONES DE LAS VARIABLES}

\begin{tabular}{|c|c|}
\hline Variable & Definición \\
\hline ANIMTOT & El número total de animales que mantiene el agricultor. \\
\hline BAJO-EMB & $\begin{array}{l}\text { Variable binaria }=1 \text { si el (los) predio(s) del agricultor se ubica en el área bajo } \\
\text { el embalse Paloma }\end{array}$ \\
\hline CAPALM & $\begin{array}{l}\text { La capacidad total de almacenamiento de agua en el predio del agricultor en } \\
\text { miles de metros cúbicos. }\end{array}$ \\
\hline COMPRA-A & $\begin{array}{l}\text { Variable binaria=1 si el agricultor compró volúmenes de agua en la } \\
\text { temporada } 1996 / 97 \text {. }\end{array}$ \\
\hline COMPRA-D & $\begin{array}{l}\text { Variable binaria }=1 \text { si el agricultor compró derechos de aprovechamiento de } \\
\text { agua entre } 1981 \text { y } 1996 .\end{array}$ \\
\hline DFAM & $\begin{array}{l}\text { Variable binaria }=1 \text { si el agricultor emplea sólo trabajadores familiares } \\
\text { (agricultor familiar) }\end{array}$ \\
\hline DPERM & $\begin{array}{l}\text { Variable binaria }=1 \text { si el agricultor dedica algún terreno a los cultivos } \\
\text { permanentes. }\end{array}$ \\
\hline DNOAG & $\begin{array}{l}\text { Variable binaria }=1 \text { si el agricultor recibe la mayoría de su ingreso de las } \\
\text { actividades no agrícolas. }\end{array}$ \\
\hline DPEQ & $\begin{array}{l}\text { Variable binaria }=1 \text { si el agricultor emplea menos de } 3 \text { trabajadores } \\
\text { permanentes y/o } 40 \text { trabajadores temporarios dentro de una temporada } \\
\text { (agricultor pequeño) }\end{array}$ \\
\hline EXP & $\begin{array}{l}\text { El número de años de experiencia agrícola del agricultor en el valle del Río } \\
\text { Limarí. }\end{array}$ \\
\hline HACULT & $\begin{array}{l}\text { El tamaño del predio en hectáreas de terreno cultivable perteneciendo c } \\
\text { arrendado por el agricultor. Donde hay más de un predio es la suma de } \\
\text { terreno cultivable. }\end{array}$ \\
\hline HAUVAEX & Hectáreas de terreno cultivable dedicado a uva de exportación. \\
\hline LAMBDA1 & $\begin{array}{l}\text { Un término de corrección de la selección de muestra para el probit censurado } \\
\text { de comprar vs. vender en el mercado spot }\end{array}$ \\
\hline LAMBDA2 & $\begin{array}{l}\text { Un término de la selección de muestra para el probit censurado de comprar } \\
\text { vs. vender en el mercado de derechos de aprovechamiento de agua. }\end{array}$ \\
\hline MULTSEC & Variable binaria $=1$ si el agricultor maneja predios en más de un sector. \\
\hline NPREDIOS & El número de predios manejados por un agricultor. \\
\hline PART-MDA & $\begin{array}{l}\text { Variable binaria }=1 \text { si el agricultor participó en el mercado de derechos de } \\
\text { aprovechamiento de agua entre } 1981 \text { y } 1996 .\end{array}$ \\
\hline PART-MS & $\begin{array}{l}\text { Variable binaria=1 si el agricultor participó en el mercado spot durante la } \\
\text { temporada } 1996 / 97 \text {. }\end{array}$ \\
\hline PORCPERM & El porcentaje de terreno cultivable dedicado a los cultivos permanentes. \\
\hline REFAG & Variable binaria=1 si el agricultor recibió sus terrenos en la reforma agraria. \\
\hline REFAGP & $\begin{array}{l}\text { Variable binaria }=1 \text { si el agricultor recibió sus terrenos en la reforma agraria y } \\
\text { se puede clasificar como un agricultor pequeño }\end{array}$ \\
\hline TANQUE & $\begin{array}{l}\text { Variable binaria }=1 \text { si el agricultor tiene por lo menos una tranque de } \\
\text { acumulación de agua ubicado en el predio. }\end{array}$ \\
\hline TECRIEGO & $\begin{array}{l}\text { Variable binaria }=1 \text { si el agricultor tiene un sistema de riego tecnificado como } \\
\text { riego por goteo o microaspersión. }\end{array}$ \\
\hline VENDIO-D & $\begin{array}{l}\text { Variable binaria=1 si el agricultor había vendido derechos de } \\
\text { aprovechamiento de agua entre } 1981 \text { y } 1996 \text {. }\end{array}$ \\
\hline VENDIO-A & $\begin{array}{l}\text { Variable binaria=1 si el agricultor había vendido agua durante la temporada } \\
1996 / 97 \text {. }\end{array}$ \\
\hline
\end{tabular}


Finalmente, en términos de la eficiencia de riego, se ve poca diferencia entre los participantes del mercado spot y los del mercado de derechos de aprovechamiento de agua. El porcentaje de tecnificación de riego es similar en ambos mercados $(28.2 \%$ vs. $33.3 \%$, respectivamente), aunque más agricultores que participan en el mercado spot tienen tranques de acumulación de agua en el predio $(50.7 \%)$ que aquellos que participan en el mercado de derechos de aprovechamiento de agua $(36.8 \%)$. La capacidad de almacenamiento de agua por hectárea también es mayor en el caso de los participantes del mercado spot (4.349 $\mathrm{m}^{3} /$ ha) que en el de los participantes del mercado de derechos de aprovechamiento de agua $\left(3.512 \mathrm{~m}^{3} / \mathrm{ha}\right)$.

\section{Modelo EMPÍrico}

La participación en los mercados spot y de derechos de aprovechamiento de agua, y las decisiones de comprar o vender, se estiman por separado mediante modelos univariados de "probit" de dos etapas. Las decisiones de comprar o vender en el mercado spot o en el mercado de derechos de aprovechamiento de agua podrían estar correlacionadas con las decisiones originales de participación. De esta forma, se estimarán las ecuaciones de la segunda etapa como "probits" censurados, dependientes de la participación. El siguiente modelo se basa en los modelos presentados por Van de Ven y Van Praag (1981) y Abdulai y Delgado (1999).

\subsection{La participación en los mercados de agua}

Un agricultor participará en el mercado spot $(\mathrm{j}=1)$ o en el mercado de derechos de aprovechamiento de agua $(\mathrm{j}=2)$ si la utilidad esperada del ingreso resultante de la participación es mayor que la de la no participación. Matemáticamente:

$$
\mathrm{EU}_{\mathrm{i}}\left(\tilde{\pi}_{\mathrm{j}}\right)=\mathrm{X}_{\mathrm{ij}}^{\prime} \mathrm{B}_{\mathrm{j}}+\varepsilon_{\mathrm{ij}}
$$

donde $\mathrm{EU}_{\mathrm{i}}\left(\tilde{\pi}_{\mathrm{j}}\right)$ es la utilidad esperada del ingreso por la participación en el mercado $\mathrm{j}$ del agricultor $\mathrm{i}, \mathrm{X}_{\mathrm{ij}}$ son vectores $(\mathrm{K}$ x 1 ) de variables exógenos que podrían influir en las decisiones de participación del agricultor, $\mathrm{i}$ y $\mathrm{B}_{\mathrm{j}}$ son vectores $(1 \mathrm{x} \mathrm{K})$ de parámetros desconocidos. Los errores $\left(\varepsilon_{\mathrm{ij}}\right)$ siguen una distribución normal estándar, i.e., $\mathrm{E}\left(\varepsilon_{\mathrm{ij}}\right)=0$ y $\mathrm{E}\left(\varepsilon_{\mathrm{ij}}^{2}\right)=1$.

La utilidad esperada del ingreso de los agricultores individuales no se observa, sino su participación o falta de participación en el mercado j. La participación/no participación del agricultor en el mercado se usa como una variable indicativa para inferir el signo de la utilidad esperada de ingreso. Se define esta variable indicativa como: 
(6)

$$
\begin{aligned}
& Z_{i j}=1 \text { iff } E_{i}\left(\tilde{\pi}_{j}\right) \geq 0 \\
& Z_{i j}=0 \text { iff } E_{i}\left(\tilde{\pi}_{j}\right)<0
\end{aligned}
$$

Por lo tanto, se puede definir la probabilidad de que un agricultor participe en el mercado j, como:

$$
\operatorname{Pr}\left\{\mathrm{Z}_{\mathrm{ij}}=1\right\}=\operatorname{Pr}\left\{\varepsilon_{\mathrm{ij}} \leq \mathrm{X}_{\mathrm{ij}}^{\prime} \mathrm{B}_{\mathrm{j}}\right\}=\Phi\left(\mathrm{X}_{\mathrm{ij}}^{\prime} \mathrm{B}_{\mathrm{j}}\right)
$$

donde $\Phi(\bullet)$ es la función de densidad cumulativa normal estándar. Así, la función de verosimilitud a maximizar para cada uno de los mercados es:

$$
\mathrm{L}=\prod_{\mathrm{Z}_{\mathrm{ij}=1}}\left[\Phi\left(\mathrm{X}_{\mathrm{ij}}^{\prime} \mathrm{B}_{\mathrm{j}}\right)\right]_{\mathrm{Z}_{\mathrm{ij}=0}}\left[1-\Phi\left(\mathrm{X}_{\mathrm{ij}}^{\prime} \mathrm{B}_{\mathrm{j}}\right)\right]
$$

Las variables $\mathrm{X}_{\mathrm{ij}}$ que influyen en la probabilidad de participación en los mercados de agua son aquellas que afectan la valoración del producto marginal del agua, la eficiencia y la seguridad de riego, los niveles de aversión al riesgo individuales y las características socioeconómicas de los agricultores.

La valoración del producto marginal del agua depende mayoritariamente del rubro de producción, el cual se puede categorizar en tres tipos: cultivos permanentes, cultivos anuales y pradera, y ganadería. Para capturar la importancia de la producción de cultivos permanentes en el modelo del mercado spot, se incluye una variable indicativa de productores de cultivos permanentes. En el modelo del mercado de derechos de aprovechamiento de agua se incluyen variables que miden el porcentaje de terreno dedicado a cultivos permanentes y el número de hectáreas dedicadas a la producción de uva de mesa de exportación. Estas variables también representan la influencia del riesgo del stock de cultivos permanentes sobre la participación en los mercados. La importancia de la ganadería se considera mediante una variable que mide la cantidad de animales que maneja el agricultor en el predio.

Para representar la eficiencia y la seguridad del riego en el predio se ocupa una variable que mide la capacidad de almacenamiento de agua en el predio en miles de metros cúbicos. Una mayor capacidad de almacenamiento de agua en el predio incrementa la probabilidad de que un agricultor participe en el mercado de agua; por lo tanto, se espera un signo positivo para el coeficiente de esta variable. Una mayor capacidad de almacenamiento aumenta la eficiencia y la seguridad del riego en el predio, permitiendo que los agricultores controlen mejor la aplicación de agua en sus terrenos.

Se supone que los agricultores muestran una aversión absoluta al riesgo decreciente si al aumentar su ingreso, éstos están dispuestos aceptar más riesgo. Por lo tanto, distintos niveles de aversión al riesgo se representan a través de variables que miden el ingreso del agricultor. Los datos sobre ingresos y deudas, recopilados mediante encuestas directas de agricultores, son poco confiables. 
Por lo tanto, se utiliza información sobre la escala de operación como una medida indirecta del ingreso.

Las variables que miden la escala de operación del agricultor incluyen el tamaño del predio, el número de predios manejados por el agricultor y si el agricultor tiene predios en más de un sector de la cuenca (p. ej. si riega con agua de más de una fuente física dentro del sistema Paloma). Se espera que los coeficientes de estas variables tengan signos positivos. Adicionalmente, en el modelo del mercado de derechos de aprovechamiento de agua se incluyen variables relacionadas más estrechamente con la escala de operación, tales como si el agricultor es pequeño, si maneja una operación familiar o si recibió su terreno en la reforma agraria. Se espera que estas variables tengan signos negativos. Es decir, que los agricultores grandes y con mayores recursos comprarán a los de menor ingreso, lo que es consistente con la teoría de repartición de riesgo.

Ciertas variables socioeconómicas también podrían influir la decisión de participar en los mercados de agua, ya que captan atributos que no se pueden medir fácilmente con otras variables. Tales variables incluyen la edad del agricultor, su nivel de educación y su experiencia en la producción agrícola en el valle. No se recopiló información en la encuesta acerca del nivel de educación del agricultor. Entre las demás variables, la edad del agricultor y su experiencia en la producción agrícola en el valle podrían estar correlacionadas. Por lo tanto, la experiencia en la producción agrícola en el valle es la única variable socioeconómica incluida en los modelos empíricos.

En el mercado de derechos de aprovechamiento de agua, una variable adicional que influye la participación es la ubicación de los predios del agricultor bajo o sobre el embalse Paloma. Específicamente, los agricultores con predios ubicados bajo el embalse Paloma podrían ser más propensos a participar en el mercado de derechos de aprovechamiento de agua debido a un área de mercado más extensa y costos de transacción menores. Sobre el embalse Paloma, hay muy poca capacidad de almacenamiento centralizado de agua, los ríos son completamente desregulados y el agua se entrega directa y secuencialmente desde los ríos a las compuertas de canal. Este sistema de distribución hace difícil traspasar los derechos de aprovechamiento de agua entre agricultores que no se ubican en el mismo canal sin altos costos de transacción.

Finalmente, para evaluar la posible interacción entre mercados se incluye en ambos modelos de participación una variable que indica si el agricultor ha participado en el otro mercado. Es posible que la probabilidad de participación en un mercado se incremente con la participación previa en el otro. Es decir, podría haber agricultores que son más propensos a vender o comprar tanto derechos de aprovechamiento de agua como volúmenes de agua. O podría ser que los agricultores que participan en el mercadospot sean significativamente distintos de aquellos que participan en el mercado de derechos de aprovechamiento de agua.

\subsection{Las decisiones de compraventa en los mercados de agua}

Dada su participación en un mercado de agua, un agricultor ofrecería o demandaría agua o derechos de aprovechamiento de agua, dependiendo si su 
precio de reserva es menor o mayor que el precio del mercado. Si el precio de reserva del agricultor es menor que el precio del mercado, el agricultor participará en el lado de oferta del mercado. Si el precio de reserva es mayor que el precio del mercado, participará en el lado de la demanda del mercado.

Se puede especificar una serie de ecuaciones para los precios de reserva y del mercado del agricultor $\mathrm{i}$ en el mercado $\mathrm{j}$ como:

$$
\mathrm{P}_{\mathrm{ij}}^{\mathrm{r}}=\mathrm{X}_{\mathrm{ij}}^{\mathrm{r}^{\prime}} \Gamma_{\mathrm{rj}}+\mathrm{u}_{\mathrm{rij}}
$$

$$
P_{\mathrm{ij}}^{\mathrm{m}}=\mathrm{X}_{\mathrm{ij}}^{\mathrm{m}^{\prime}} \Gamma_{\mathrm{mj}}+\mathrm{u}_{\mathrm{mij}}
$$

donde $P_{i j}^{r}$ es el precio de reserva del agricultor i en el mercado $j . \mathrm{P}_{\mathrm{ij}}^{\mathrm{m}}$ es el precio que ofrece en el mercado $\mathrm{j}$, y $\mathrm{X}_{\mathrm{ij}}^{\mathrm{r}}$ y $\mathrm{X}_{\mathrm{ij}}^{\mathrm{m}}$ son vectores ( $\mathrm{K}$ X 1) de variables explicativas que influyen en los precios de reserva y del mercado. $\Gamma_{\mathrm{rj}}$ y $\Gamma_{\mathrm{mj}}$ son vectores de parámetros desconocidos.

Se define la propensión a comprar o vender agua, $\mathrm{P}_{\mathrm{i} 1}^{*}, \mathrm{y}$ a comprar o vender derechos de aprovechamiento de agua, $\mathrm{P}_{\mathrm{i} 2}^{*}$, como:

$$
P_{i j}^{*}=X_{i j}^{\prime} \Gamma_{j}+v_{i j}
$$

donde $P_{i j}^{*}=P_{i j}^{r}-P_{i j}^{m}, X_{i j}^{\prime} \Gamma_{j}=X_{i j}^{r} \Gamma_{y j}-X_{i j}^{m} \Gamma_{m j} \quad y \quad v_{i j}=u_{r i j}-u_{m i j} \cdot$

Semejante a las decisiones de la participación en el mercado de agua, no se observa la propensión de un agricultor a ser un oferente, o un demandante de agua o de los derechos de aprovechamiento de agua, sino su actividad en el mercado. Ocupando esta información se pueden construir variables indicadoras censuradas, dependientes de la participación:

$$
\begin{aligned}
& \left(\mathrm{Y}_{\mathrm{ij}}=1 \mid \mathrm{Z}_{\mathrm{ij}}=1\right) \text { iff } \mathrm{P}_{\mathrm{ij}}^{*}>0 \\
& \left(\mathrm{Y}_{\mathrm{ij}}=0 \mid \mathrm{Z}_{\mathrm{ij}}=1\right) \text { iff } \mathrm{P}_{\mathrm{ij}}^{*} \leq 0
\end{aligned}
$$

donde $Y_{i j}=1$ si el agricultor es un demandante en el mercado $j$ e $Y_{i j}=0$ si el agricultor es un oferente.

La censura de las variables dependientes a ser utilizados en los modelos de probit resultará en estimaciones sesgadas e inconsistentes si existe correlación entre los términos de error de los modelos de participación y sus correspondientes modelos de oferta/demanda. Para corregir este sesgo potencial, se emplea el método introducido por Van de Ven y Van Praag (1981), el cual adapta el estimador de dos etapas al modelo de probit censurado. Con este propósito se redefine (10) como:

$$
E\left[P_{i j}^{*} \mid X_{i j}, E U_{i j}\left(\tilde{\pi}_{i j}\right)>0\right]=X_{i j}^{\prime} \Gamma_{j}+E\left[v_{i j} \mid X_{i j}, E U_{i j}\left(\tilde{\pi}_{i j}\right)>0\right]
$$


Suponiendo que $v_{\mathrm{ij}}$ y $\varepsilon_{\mathrm{ij}}$ siguen una distribución normal estándar bivariada con coeficiente de correlación $\rho$, se obtiene:

$$
\mathrm{E}\left[v_{\mathrm{ij}} \mid \mathrm{X}_{\mathrm{ij}}, \mathrm{EU}_{\mathrm{i}}\left(\tilde{\pi}_{\mathrm{ij}}\right) \geq 0\right]=\rho \lambda_{\mathrm{ij}}
$$

donde $\lambda_{\mathrm{ij}}$ es el inverso de la razón de Mill, calculado de la regresión probit del mercado j. Combinando (12) y (13), se obtiene:

$$
P_{i j}^{*}=X_{i j}^{\prime} \Gamma_{j}+\rho \lambda_{i j}+\tilde{v}_{i j}
$$

donde $\mathrm{E}\left(\tilde{\mathrm{v}}_{\mathrm{ij}} \mid \mathrm{EU} \mathrm{i}_{\mathrm{i}}\left(\tilde{\pi}_{\mathrm{ij}}\right) \geq 0\right)=0$ y $\mathrm{E}\left(\tilde{\mathrm{v}}_{\mathrm{ij}}^{2} \mid \mathrm{EU}_{\mathrm{i}}\left(\tilde{\pi}_{\mathrm{ij}}\right) \geq 0\right)=\tau_{\mathrm{ij}}^{2}$.

Para identificar los parámetros de la regresión probit de segunda etapa, se debe transformar la ecuación para que el término de error tenga una varianza unitaria. Dividiendo la ecuación (14) por $\tau_{\mathrm{ij}}$ se obtiene el modelo corregido:

$$
\mathrm{P}_{\mathrm{ij}}^{* *}=\mathrm{X}_{\mathrm{ij}}^{* \prime} \Gamma_{\mathrm{j}}+\rho \lambda_{\mathrm{ij}}^{*}+v_{\mathrm{ij}}^{*}
$$

donde $*$ indica que la variable se ha dividido por $\tau_{\mathrm{ij}}$.

La probabilidad de que un agricultor compre agua dado que este participa en mercado j es:

$$
\operatorname{Pr}\left\{\mathrm{Y}_{\mathrm{ij}}=1 \mid \mathrm{Z}_{\mathrm{ij}}=1\right\}=\Phi_{\mathrm{v}}\left(\mathrm{X}_{\mathrm{ij}}^{*^{\prime \prime}} \Gamma_{\mathrm{j}}+\rho \lambda_{\mathrm{ij}}^{*}\right)
$$

La función de verosimilitud a maximizar tiene la misma forma general que (8).

Las variables $\mathrm{X}_{\mathrm{ij}}$ influirán la probabilidad de ser un oferente o un demandante de agua o de derechos de aprovechamiento de agua. Como está planteado en el modelo conceptual, aunque habrá traspasos de agua y de derechos de aprovechamiento de agua basados en las diferencias en el valor del producto marginal de agua, también se generarán traspasos basados en las diferencias en la eficiencia de riego y en el deseo de repartir riesgo entre agricultores con distintas tolerancias al riesgo. En resumen, en ambos mercados los agricultores con sistemas de riego menos eficientes venderán a aquellos con sistemas más eficientes, y los agricultores menos tolerantes al riesgo venderán a los más tolerantes. Además, ciertos factores socioeconómicos también podrían influir la probabilidad de ser un oferente o un demandante en los mercados.

La eficiencia de riego se representa en ambos modelos por medio de una variable que mide la capacidad de almacenamiento de agua en el predio. Además, el modelo del mercado de derechos de aprovechamiento de agua contiene dos variables adicionales: la existencia de un sistema de riego tecnificado y la existen- 
cia de tranques de acumulación de agua en el predio. Se esperan signos positivos para los coeficientes de todas estas variables.

El efecto del nivel de aversión al riesgo se incorpora la escala de operación como una proxy para el ingreso, como se hizo en los modelos de participación. Para representar a los agricultores grandes de mayores recursos se incluye una variable que mide el tamaño del (de los) predio (s). Además, en el caso del mercado spot, se agregan como variables el número de predios que tiene el agricultor y si éste recibe ingresos adicionales de actividades no-agrícolas. Se esperan signos positivos para los coeficientes de estas variables.

Para representar a los agricultores de bajos ingresos se incluye en ambos modelos una variable que identifica a los agricultores que consiguieron sus terrenos mediante la reforma agraria y que todavía se pueden clasificar como pequeños agricultores. Estos agricultores tienden a ser de los más pobres en la cuenca del Limarí. Además, en la regresión del mercado spot se incluye una variable que indica si el agricultor había vendido derechos de aprovechamiento de agua anteriormente a su participación en dicho mercado, ya que la mayoría de los agricultores encuestados indicaron que habían vendido derechos de aprovechamiento de agua para financiar inversiones o pagar deudas. Se esperan signos negativos para los coeficientes de estas variables.

Finalmente, se incluye en ambos modelos una variable que mide la experiencia del agricultor en producción agrícola en el valle Limarí como característica socioeconómica y el inverso de la razón de Mill.

\section{Los Resultados}

\subsection{La participación en los mercados de agua}

Los resultados de los modelos de la probabilidad de que un agricultor participare en algún mercado de agua señalan una dicotomía entre los dos mercados (vea Cuadros 3 y 4). El mercado spot está dominado por agricultores de cultivos anuales, y el mercado de derechos de aprovechamiento de agua está dominado por agricultores de cultivos permanentes de alto valor, como es la uva de mesa de exportación. También, la probabilidad de participación en el mercado de derechos de aprovechamiento de agua se incrementa significativamente con el porcentaje de superficie cultivable dedicada a uva de mesa de exportación, aunque el porcentaje de superficie cultivable dedicada a cultivos permanentes en general no es significativo. Los resultados verifican la importancia del riesgo adicional que enfrentan los productores de cultivos permanentes de alto valor en sus decisiones de participación en los mercados de agua.

En el mercado de derechos de aprovechamiento de agua, la ubicación de los predios de un agricultor bajo el embalse Paloma incrementa la probabilidad de participación en el mercado de derechos de aprovechamiento de agua. Este es un resultado importante, porque la mayoría de las cuencas en Chile tiene una infraestructura de distribución de agua similar a aquella del área sobre el embalse Paloma 
en el valle del Río Limarí. En estas redes de distribución se hacen difíciles los traspasos de agua y de derechos de aprovechamiento de agua debido a los altos costos de transacción. La significancia de esta variable como un determinante de la participación en el mercado de derechos de aprovechamiento de agua muestra que una infraestructura adecuada y flexible es esencial para fomentar un traspaso eficiente de los derechos de aprovechamiento de agua desde los usos de bajo valor a usos de alto valor.

CUADRO 3

LOS RESULTADOS DEL MODELO PROBIT CENSURADO: LA PARTICIPACION EN EL MERCADO SPOT Y LA DECISION DE COMPRAR O VENDER AGUA

Participación en el mercado spot

Variable dependiente: PART-MS

\begin{tabular}{lccc}
\hline Variable explicativa & $\begin{array}{c}\text { Coeficiente } \\
\text { estimado }\end{array}$ & $\begin{array}{c}\text { Error } \\
\text { estándar }\end{array}$ & $\begin{array}{c}\text { Estadístico } \\
\mathrm{t}\end{array}$ \\
\hline DPERM* $^{*}$ & $-0,4210$ & 0,1999 & $-2,106$ \\
ANIMTOT* $^{*}$ & $-0,5362$ & 0,0026 & $-2,095$ \\
PART-MDA & $-0,2762$ & 0,2130 & $-1,297$ \\
MULTSEC ** $^{*}$ & 0,8079 & 0,4367 & 1,850 \\
CAPALM* & 0,0330 & 0,0166 & 1,983 \\
HACULT & 0,0017 & 0,0025 & 0,692 \\
EXP & $-0,0261$ & 0,0068 & $-0,384$ \\
NPREDIOS & 0,1723 & 0,1052 & 1,637 \\
CONSTANT & $-0,3244$ & 0,2620 & $-1,238$ \\
\hline
\end{tabular}

Número de observaciones: 199

Chi-cuadrado: 24,37 con 8 g. de 1 .

$\%$ Predicciones correctas: $68,34 \%$

Comprar o vender agua

Variable dependiente: COMPRA-A

\begin{tabular}{lccc}
\hline Variable explicativa & $\begin{array}{c}\text { Coeficiente } \\
\text { estimado }\end{array}$ & $\begin{array}{c}\text { Error } \\
\text { estándar }\end{array}$ & $\begin{array}{c}\text { Estadístico } \\
\mathrm{t}\end{array}$ \\
\hline DNOAG* $^{*}$ & $-1,1158$ & 0,1984 & $-2,244$ \\
VENDIO-A* $^{*}$ & $-1,3083$ & 0,2326 & $-2,244$ \\
CAPALM & 0,0747 & 0,0303 & 0,986 \\
REFAGP* & $-1,1696$ & 0,1956 & $-2,382$ \\
EXP & $-0,0015$ & 0,0059 & $-0,025$ \\
HACULT** & $-0,0111$ & 0,0058 & $-1,907$ \\
NPREDIOS & 0,1437 & 0,1291 & 1,113 \\
CONSTANTE & 0,3934 & 0,3939 & 0,999 \\
LAMBDA1 & $-0,1292$ & 0,3145 & $-0,411$ \\
\hline
\end{tabular}

Número de observaciones: 65

Chi-cuadrado: 27,61 con 8 g. de 1.

$\%$ Predicciones correctas: $76,92 \%$

*significativo al nivel $5 \%$

**significativo al nivel $10 \%$ 


\section{CUADRO 4}

LOS RESULTADOS DEL MODELO PROBIT CENSURADO: PARTICIPACION EN EL MERCADO DE DERECHOS DEAPROVECHAMIENTO DE AGUA Y LA DECISION DE COMPRAR O VENDER

Participación en el mercado de derechos de aprovechamiento de agua

Variable dependiente: PART-MDA

\begin{tabular}{lccc}
\hline Variable explicativa & $\begin{array}{c}\text { Coeficiente } \\
\text { estimado }\end{array}$ & $\begin{array}{c}\text { Error } \\
\text { estándar }\end{array}$ & $\begin{array}{c}\text { Estadístico } \\
\mathrm{t}\end{array}$ \\
\hline HACULT & 0,0014 & 0,0021 & 0,671 \\
MULTSEC* $^{*}$ & 0,9874 & 0,4098 & 2,409 \\
CAPALM** & 0,0282 & 0,0144 & 1,954 \\
BAJO-EMB* & 0,7530 & 0,2157 & 3,491 \\
PART-MS & $-0,3597$ & 0,2194 & $-1,639$ \\
PORCPERM & 0,3983 & 0,3185 & 1,251 \\
HAUVAEX* & 0,8804 & 0,3641 & 2,418 \\
EXP & 0,0016 & 0,0627 & 0,261 \\
ANIMTOT & $-0,0020$ & 0,0191 & $-1,068$ \\
REFAG** & 0,3240 & 0,1824 & 1,777 \\
DFAM** & 0,4963 & 0,2618 & 1,895 \\
DPEQ & 0,0111 & 0,2152 & 0,051 \\
NPREDIOS & 0,0579 & 0,1005 & 0,577 \\
CONSTANTE & $-1,7660$ & 0,3811 & $-4,634$ \\
\hline
\end{tabular}

Número de observaciones: 301

Chi-cuadrado: 42,62 con 13 g. de 1.

$\%$ Predicciones correctas: $76,08 \%$

Comprar o vender derechos de aprovechamiento de agua

Variable dependiente: COMPRA-D

\begin{tabular}{lccc}
\hline Variable explicativa & $\begin{array}{c}\text { Coeficiente } \\
\text { estimado }\end{array}$ & $\begin{array}{c}\text { Error } \\
\text { estándar }\end{array}$ & $\begin{array}{c}\text { Estadístico } \\
\mathrm{t}\end{array}$ \\
\hline TECRIEGO*** & 1,3173 & 0,4287 & 3,073 \\
TANQUE*** & 1,1776 & 0,4514 & 2,609 \\
CAPALM & 0,0085 & 0,0268 & 0,318 \\
HACULT & 0,0010 & 0,0057 & 0,170 \\
REFAGP* & $-1,4451$ & 0,6474 & $-2,232$ \\
EXP & $-0,0140$ & 0,0117 & $-1,197$ \\
LAMBDA2 & $-0,4701$ & 0,3180 & $-1,478$ \\
\hline
\end{tabular}

Número de observaciones: 83

Chi cuadrado: 54,653

$\%$ Predicciones correctas: $83,75 \%$

$*$ significativo al nivel $5 \% \quad * *$ significativo al nivel $10 \%$

*** significativo al nivel $1 \%$ 
A pesar de sus diferencias, los mercados también son similares en cierta medida. En ambos mercados, la capacidad de almacenamiento de agua en el predio influye positivamente en la probabilidad de participación. La escala de operación también es un determinante importante de la participación en ambos mercados. Aunque el tamaño del predio y el número de predios que maneja el agricultor no son significativos en ninguno de los modelos, hay una representación importante de agricultores con parcelas en más de un sector en ambos mercados. Además, el hecho de ser un agricultor familiar o de la reforma agraria influye positivamente en la participación en el mercado de derechos de aprovechamiento de agua, indicando quizás que el papel de la repartición del riesgo, basado en distintos niveles de aversión al riesgo entre agricultores, es más importante en este mercado que en el mercado spot. Finalmente, la participación de un agricultor en un mercado no influye su probabilidad de participación en el otro mercado, siendo los coeficientes de las variables de participación no significativos en ambos modelos.

\subsection{Comprar o vender}

La eficiencia de riego es un determinante importante de la probabilidad de que un agricultor venderá o comprará, pero solamente en el mercado de derechos de aprovechamiento de agua. Se observa este resultado en los coeficientes significativos y positivos de la existencia de tranques de acumulación de agua en el predio y de riego tecnificado en el modelo del mercado de derechos de aprovechamiento de agua (vea Cuadro 4). Cabe señalar que diferencias en la capacidad de almacenamiento de agua en el predio no influyen en la probabilidad de ser un oferente o un demandante en ninguno de los mercados (vea Cuadros 3 y 4 ).

Los agricultores de bajos ingresos y de liquidez limitada tienden a ser vendedores en los mercados de agua, lo que es consistente con la teoría de repartición de riesgo. Este resultado se demuestra por los coeficientes negativos y significativos de las variables indicativas de pequeños agricultores de la reforma agraria en ambos mercados y de la variable que indica si el agricultor había vendido derechos de aprovechamiento de agua anteriormente a su participación en el mercado spot.

Sin embargo, los agricultores con más terreno y/o aquellos que reciben ingreso no agrícola también tienden a ser vendedores en el mercado spot. Estos resultados contradicen la hipótesis de que a mayor ingreso, la probabilidad de ser un vendedor disminuye en ambos mercados. Dicho resultado podría deberse a que existen economías de escala en el mercadospot, las que hacen más conveniente para los agricultores grandes el vender agua en años secos para cubrir sus costos fijos en vez de intentar establecer una plantación rentable con abastecimiento de agua escaso e inseguro y altos precios para agua en el mercado. También sugiere que los compradores en el mercado spot podrían ser agricultores medianos, sin restricciones de liquidez, que se dedican completamente a la producción agrícola. 


\section{ConCLusión}

Esta investigación examinó el efecto de la incertidumbre y del riesgo sobre los movimientos de recursos hídricos en un mercado de agua en el sector agrícola chileno. Se planteó la hipótesis de que el valor del producto marginal del agua es una medida inadecuada del valor del agua en la agricultura debido a la incertidumbre que rodea la producción agrícola. También se planteó la hipótesis de que los efectos de esta incertidumbre sobre las decisiones de los agricultores difieren dependiendo de los distintos riesgos que éstos enfrentan y de su tolerancia al riesgo.

Los resultados muestran interesantes patrones de comercio en los mercados de agua de la cuenca del Río Limarí. Los agricultores para quienes un déficit de agua es más riesgoso para la producción tienden a participar menos en el mercado spot y más en el mercado de los derechos de aprovechamiento de agua donde tienden a ser compradores. Por esta razón, se suele observar que los productores de cultivos anuales dominan los mercados spot, mientras que los productores de cultivos permanentes tienden a participar en los mercados de derechos de aprovechamiento de agua. Los agricultores de ingresos bajos y aquellos que enfrentan importantes restricciones de liquidez tienden a vender agua o vender derechos de aprovechamiento de agua, de acuerdo con la hipótesis de una aversión absoluta decreciente al riesgo y su preferencia por un ingreso cierto, a través de la venta de agua o de derechos de aprovechamiento, en lugar de un ingreso incierto mediante la producción regada.

La manutención de un excedente de derechos de aprovechamiento de agua, o compras adicionales, podrían parecer como acaparamiento o un uso no benéfico del agua a los políticos. Sin embargo, esto refleja claramente un comportamiento racional de algunos productores para reducir el riesgo. Dichos derechos proveen un tipo de seguro contra la sequía para los agricultores que enfrentan altos riesgos. Aunque no se les utilizan durante los años con agua abundante o adecuado, estos derechos son críticos durante las temporadas de déficit de agua.

El uso de derechos de aprovechamiento de agua como una protección contra el riesgo de una sequía no implica que el agua se asigne ineficientemente. Cuando se incorpora el riesgo y la incertidumbre en la valoración de los derechos de aprovechamiento de agua, se redefine la eficiencia de la asignación del agua agregando el criterio de una repartición eficaz del riesgo entre los agricultores. La política de patentes de no uso, dirigida a mejorar la eficiencia de la asignación de los derechos de aprovechamiento de agua, por lo tanto, reduciría la capacidad del mercado para repartir el riesgo eficientemente en el sector agrícola. Por ende, cualquier política nacional dirigida hacia el uso del agua en el sector agrícola chileno debe tomar en cuenta estos factores importantes que hasta el momento se han ignorado. 


\section{REFERENCIAS}

Abdulai, A. y C. L. Delgado (1999), "Determinants of Nonfarm Earnings on Farm-Based Husbands and Wives in Northern Ghana", American Journal of Agricultural Economics 81: 117-130.

Bauer, C. (1998), "Against the Current? Privatization, Water Markets and the State in Chile", Boston: Kluwer Academic Press.

Beare, S., R. Bell y B. S. Fisher (1998), "Determining the Value of Water: The Role of Risk, Infrastructure Constraints, and Ownership", American Journal of Agricultural Economics 80: 916-940.

Colby- Saliba, B. (1990), “Transactions Costs and Efficiency in Western Water Allocation", American Journal of Agricultural Economics 72: 1184-1192.

Colby-Saliba, B. y D. Bush (1987), Water Markets in Theory and Practice, Boulder: Westview Press.

Heckman, J. (1976), "The Common Structure of Statistical Models of Truncation, Sample Selection and Limited Dependent Variables and a Simple Estimator for Such Models", Annals of Economic and Social Measurement 5: 475-492.

Heckman, J. (1979), "Sample Selection Bias as a Specification Error", Econometrica 47: 153-161.

Howitt, R. E. (1998), "Spot Prices, Option Prices, and Water Markets: An Analysis of Emerging Markets in California. Markets for Water: Potential and Performance", K. William Easter, M. Rosegrant and A. Dinar, eds. Boston: Kluwer Academic Press.

Maddala, G.S. (1998), Limited Dependent and Qualitative Variables in Econometrics, Cambridge: Cambridge University Press.

Rosegrant, M. y H. Binswanger (1994), "Markets in Tradable Water Rights: Potential for Efficiency Gains in Developing Country Water Resource Allocation", World Development 22: 1613-25.

Van de Ven, W. y B. Van Praag (1981), "The Demand for Deductibles in Private Health Insurance: A Probit Model with Sample Selection”, Journal of Econometrics 17: $229-252$. 


\section{APENDICE I \\ EL PROGRAMA DE OPTIMIZACION Y LAS CONDICIONES DE COMPLEMENTARY SLACKNESS}

$$
\begin{aligned}
& \max _{\alpha} \sum_{\mathrm{i}=1}^{\mathrm{N}} \mathrm{V}_{\mathrm{i}}\left(\tilde{\pi}_{\mathrm{i}}\right)=\sum_{\mathrm{i}=1}^{\mathrm{N}} \sum_{\mathrm{t}=0}^{\mathrm{T}} \beta_{\mathrm{t}} \mathrm{V}_{\mathrm{it}}\left(\tilde{\pi}_{\mathrm{i}}\right)+\beta_{\mathrm{T}+1} \mathrm{~V}_{\mathrm{iT}+1}\left(\pi_{\mathrm{iT}+1}\right) \\
& \text { s.t. } \\
& \forall \mathrm{i}, \mathrm{t} \\
& \overline{\mathrm{L}}_{\mathrm{it}}=\mathrm{L}_{\mathrm{it}}^{\mathrm{I}}+\mathrm{F}_{\mathrm{it}} \quad\left(\delta_{\mathrm{it}}\right) \\
& {\left[\mathrm{E}\left(\widetilde{\omega}_{\mathrm{t}} \mathrm{K}_{\mathrm{it}}^{\mathrm{R}}\right)+\tau_{\mathrm{n}} \sigma_{\mathrm{w}}\right] \geq \mathrm{W}_{\mathrm{it}}^{\mathrm{I}}+\mathrm{W}_{\mathrm{it}}^{\mathrm{S}}-\mathrm{W}_{\mathrm{it}}^{\mathrm{B}} \quad\left(\gamma_{\mathrm{it}}\right)} \\
& \mathrm{K}_{\mathrm{it}}^{\mathrm{R}} \equiv \mathrm{K}_{\mathrm{it}-1}^{\mathrm{R}}+\mathrm{R}_{\mathrm{it}}^{\mathrm{B}}-\mathrm{R}_{\mathrm{it}}^{\mathrm{S}} \quad\left(\lambda_{\mathrm{it}}\right) \\
& \mathrm{K}_{\mathrm{it}}^{\mathrm{P}}=\theta \mathrm{K}_{\mathrm{it}-1}^{\mathrm{P}}-\mathrm{R}_{\mathrm{it}}+\mathrm{Pl}_{\mathrm{it}} \quad\left(\rho_{\mathrm{it}}\right) \\
& \alpha=\left(\mathrm{R}_{\mathrm{it}}^{\mathrm{S}}, \mathrm{R}_{\mathrm{it}}^{\mathrm{B}}, \mathrm{W}_{\mathrm{it}}^{\mathrm{I}}, \mathrm{L}_{\mathrm{it}}^{\mathrm{I}}, \mathrm{W}_{\mathrm{it}}^{\mathrm{B}}, \mathrm{W}_{\mathrm{it}}^{\mathrm{S}}, \mathrm{K}_{\mathrm{it}}^{\mathrm{P}}\right)
\end{aligned}
$$

El programa de optimización completa es:

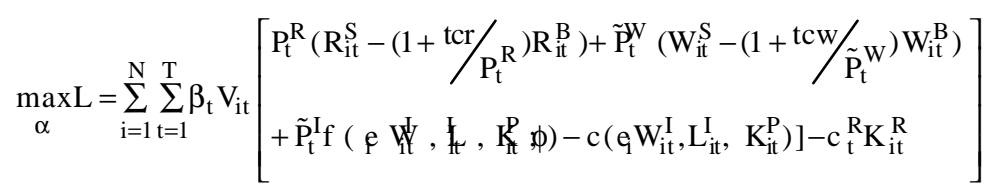

$$
\begin{aligned}
& +\sum_{\mathrm{i}=1}^{\mathrm{N}} \beta_{\mathrm{T}+1} \mathrm{~V}_{\mathrm{iT}+1}\left(\mathrm{P}_{\mathrm{T}+1}^{\mathrm{R}} \mathrm{K}_{\mathrm{iT}+1}^{\mathrm{r}}\right) \\
& +\sum_{\mathrm{i}=1}^{\mathrm{N}} \delta_{\mathrm{it}}\left(\overline{\mathrm{L}}_{\mathrm{it}}-\mathrm{L}_{\mathrm{it}}^{\mathrm{I}}-\mathrm{F}_{\mathrm{it}}\right) \\
& +\sum_{i=1}^{N} \gamma_{i t}\left(E\left(\tilde{\omega}_{t} K_{i t}^{\mathrm{P}}\right)+\tau_{n} \sigma_{w}-W_{i t}^{I}-W_{i t}^{S}+W_{i t}^{B}\right) \\
& +\sum_{i=1}^{N} \lambda_{i t}\left(K_{i t}^{R}-K_{i t-1}^{R}+R_{i t}^{B}-R_{i t}^{S}\right) \\
& +\sum_{\mathrm{i}=1}^{\mathrm{N}} \rho_{\mathrm{it}}\left(\mathrm{K}_{\mathrm{it}}^{\mathrm{P}}-\theta \mathrm{K}_{\mathrm{t}-1}^{\mathrm{P}}-\mathrm{Pl}_{\mathrm{it}}+\mathrm{R}_{\mathrm{it}}\right)
\end{aligned}
$$


Las condiciones de complementary slackness son:

$$
\begin{array}{ll}
\frac{\partial \mathrm{L}}{\partial \mathrm{W}_{\mathrm{it}}^{\mathrm{S}}} \leq 0 & \mathrm{~W}_{\mathrm{it}}^{\mathrm{S}} \frac{\partial \mathrm{L}}{\partial \mathrm{W}_{\mathrm{it}}^{\mathrm{S}}}=0 \\
\frac{\partial \mathrm{L}}{\partial \mathrm{W}_{\mathrm{it}}^{\mathrm{B}}} \geq 0 & \mathrm{~W}_{\mathrm{it}}^{\mathrm{B}} \frac{\partial \mathrm{L}}{\partial \mathrm{W}_{\mathrm{it}}^{\mathrm{B}}}=0 \\
\frac{\partial \mathrm{L}}{\partial \mathrm{W}_{\mathrm{it}}^{\mathrm{I}}}=0 & \\
\frac{\partial \mathrm{L}}{\partial \mathrm{R}_{\mathrm{it}}^{\mathrm{S}}} \geq 0 & \mathrm{R}_{\mathrm{it}}^{\mathrm{S}} \frac{\partial \mathrm{L}}{\partial \mathrm{R}_{\mathrm{it}}^{\mathrm{S}}}=0 \\
\frac{\partial \mathrm{L}}{\partial \mathrm{R}_{\mathrm{it}}^{\mathrm{B}}} \leq 0 & \mathrm{R}_{\mathrm{it}}^{\mathrm{B}} \frac{\partial \mathrm{L}}{\partial \mathrm{R}_{\mathrm{it}}^{\mathrm{B}}}=0 \\
\frac{\partial \mathrm{L}}{\partial \mathrm{K}_{\mathrm{it}}^{\mathrm{R}}}=0 & \\
\frac{\partial \mathrm{L}}{\partial \mathrm{K}_{\mathrm{it}}^{\mathrm{P}}}=0 & \\
\frac{\partial \mathrm{L}}{\partial \mathrm{L}_{\mathrm{it}}^{\mathrm{I}}}=0 & \\
\frac{\partial \mathrm{L}}{\partial \mathrm{K}_{\mathrm{iT}}^{\mathrm{R}}+1}=0 & \\
\frac{\partial \mathrm{L}}{\partial \mathrm{K}_{\mathrm{iT}+1}^{\mathrm{P}}}=0 & \\
\frac{\partial \mathrm{L}}{\lambda_{\mathrm{it}}}=0 & \\
\frac{\partial \mathrm{L}}{\partial \mathrm{\gamma}_{\mathrm{it}}} \geq 0 & \gamma_{\mathrm{it}} \frac{\partial \mathrm{L}}{\partial \gamma_{\mathrm{it}}}=0 \\
\frac{\partial \mathrm{L}}{2} &
\end{array}
$$


APENDICE II

\section{LAS CONDICIONES DE PRIMER ORDEN}

(1) $\frac{\partial \mathrm{L}}{\partial \mathrm{W}_{\mathrm{it}}^{\mathrm{S}}}=\beta_{\mathrm{t}} \mathrm{EV}_{\mathrm{it}}^{\prime}\left(\tilde{\pi}_{\mathrm{it}}\right) \tilde{\mathrm{P}}_{\mathrm{t}}^{\mathrm{W}}-\gamma_{\mathrm{it}} \leq 0 \quad \forall \mathrm{i}, \mathrm{t}$

(2) $\frac{\partial \mathrm{L}}{\partial \mathrm{W}_{\mathrm{it}}^{\mathrm{B}}}=-\beta_{\mathrm{t}} \mathrm{EV}_{\mathrm{it}}^{\prime}\left(\tilde{\pi}_{\mathrm{it}}\right) \tilde{\mathrm{P}}_{\mathrm{t}}^{\mathrm{W}}-\gamma_{\mathrm{it}} \leq 0 \quad \forall \mathrm{i}, \mathrm{t}$

(3) $\frac{\partial \mathrm{L}}{\partial \mathrm{W}_{\mathrm{it}}^{\mathrm{I}}}=\beta_{\mathrm{t}} \mathrm{E}\left\{\mathrm{V}_{\mathrm{it}}^{\prime}\left(\tilde{\pi}_{\mathrm{it}}\right)\left[\tilde{\mathrm{P}}_{\mathrm{t}}^{\mathrm{I}} \frac{\partial \mathrm{f}(\bullet)}{\partial \mathrm{e}_{\mathrm{i}} \mathrm{W}_{\mathrm{it}}^{\mathrm{I}}}-\frac{\partial \mathrm{c}(\bullet)}{\partial \mathrm{e}_{\mathrm{i}} \mathrm{W}_{\mathrm{it}}^{\mathrm{I}}}\right] \mathrm{e}_{\mathrm{i}}\right\}-\gamma_{\mathrm{it}}=0 \quad \forall \mathrm{i}, \mathrm{t}$

(4) $\frac{\partial \mathrm{L}}{\partial \mathrm{R}_{\text {it }}^{\mathrm{S}}}=\beta_{\mathrm{t}} \mathrm{EV} \mathrm{V}_{\mathrm{it}}^{\prime}\left(\tilde{\pi}_{\mathrm{it}}\right) \mathrm{P}_{\mathrm{t}}^{\mathrm{R}}-\lambda_{\mathrm{it}} \geq 0 \quad \forall \mathrm{i}, \mathrm{t}$

(5) $\frac{\partial \mathrm{L}}{\partial \mathrm{R}_{\mathrm{it}}^{\mathrm{B}}}=-\beta_{\mathrm{t}} \mathrm{EV}_{\mathrm{it}}^{\prime}\left(\tilde{\pi}_{\mathrm{it}}\right) \mathrm{P}_{\mathrm{t}}^{\mathrm{R}}+\lambda_{\mathrm{it}} \leq 0 \quad \forall \mathrm{i}, \mathrm{t}$

(6) $\frac{\partial \mathrm{L}}{\partial \mathrm{K}_{\mathrm{it}}^{\mathrm{R}}}=\beta_{\mathrm{t}} \mathrm{EV}_{\mathrm{it}}^{\prime}\left(\tilde{\pi}_{\mathrm{it}}\right)\left[-\mathrm{c}_{\mathrm{t}}^{\mathrm{R}}\right]+\mathrm{E}\left(\tilde{\omega}_{\mathrm{t}}\right) \gamma_{\mathrm{it}}-\lambda_{\mathrm{it}}+\lambda_{\mathrm{it}+1}=0 \quad \forall \mathrm{i}, \mathrm{t}$

(7) $\frac{\partial \mathrm{L}}{\partial \mathrm{K}_{\mathrm{it}}^{\mathrm{P}}}=\beta_{\mathrm{t}} \mathrm{EV} V_{\mathrm{it}}^{\prime}\left(\tilde{\pi}_{\mathrm{it}}\right)\left[\tilde{\mathrm{P}}_{\mathrm{t}}^{\mathrm{I}} \frac{\partial \mathrm{f}(\bullet)}{\partial \mathrm{K}_{\mathrm{it}}^{\mathrm{P}}}-\frac{\partial \mathrm{c}(\bullet)}{\partial \mathrm{K}_{\mathrm{it}}^{\mathrm{P}}}\right]-\rho_{\mathrm{it}}+\theta \rho_{\mathrm{it}+1}=0 \quad \forall \mathrm{i}, \mathrm{t}$

(8) $\frac{\partial \mathrm{L}}{\partial \mathrm{L}_{\mathrm{it}}^{\mathrm{I}}}=\beta_{\mathrm{t}} \mathrm{EV}_{\mathrm{it}}^{\prime}\left(\tilde{\pi}_{\mathrm{it}}\right)\left[\tilde{\mathrm{P}}_{\mathrm{t}}^{\mathrm{I}} \frac{\partial \mathrm{f}(\bullet)}{\partial \mathrm{L}_{\mathrm{it}}^{\mathrm{I}}}-\frac{\partial \mathrm{c}(\bullet)}{\partial \mathrm{L}_{\mathrm{it}}^{\mathrm{I}}}\right]-\delta_{\mathrm{it}}=0 \quad \forall \mathrm{i}, \mathrm{t}$

(9) $\frac{\partial \mathrm{L}}{\partial \mathrm{K}_{\mathrm{iT}+1}^{\mathrm{R}}}=\beta_{\mathrm{t}+1} \mathrm{EV}_{\mathrm{it}}^{\prime}\left(\tilde{\pi}_{\mathrm{iT}+1}\right) \mathrm{P}_{\mathrm{T}+1}^{\mathrm{R}}+\lambda_{\mathrm{iT}}=0 \quad \forall \mathrm{i}, \mathrm{t}$

(10) $\frac{\partial \mathrm{L}}{\partial \mathrm{K}_{\mathrm{i}+1}^{\mathrm{P}}}=\beta_{\mathrm{T}+1} \mathrm{EV}_{\mathrm{it}}^{\prime}\left(\tilde{\pi}_{\mathrm{iT}+1}\right) \mathrm{P}_{\mathrm{T}+1}+\theta \rho_{\mathrm{iT}}=0 \quad \forall \mathrm{i}, \mathrm{t}$

(11) $\frac{\partial \mathrm{L}}{\lambda_{\text {it }}}=\mathrm{K}_{\mathrm{it}}^{\mathrm{R}}-\mathrm{K}_{\mathrm{it}-1}^{\mathrm{R}}-\mathrm{W}_{\mathrm{it}}^{\mathrm{B}}+\mathrm{W}_{\mathrm{it}}^{\mathrm{S}}=0 \quad \forall \mathrm{i}, \mathrm{t}$

(12) $\frac{\partial \mathrm{L}}{\partial \gamma_{\mathrm{it}}}=\mathrm{E}\left(\tilde{\omega}_{\mathrm{t}}\right) \mathrm{K}_{\mathrm{it}}^{\mathrm{R}}+\tau_{\eta_{\mathrm{i}}} \sigma_{\omega}-\mathrm{W}_{\mathrm{it}}^{\mathrm{I}}+\mathrm{W}_{\mathrm{it}}^{\mathrm{S}}-\mathrm{W}_{\mathrm{it}}^{\mathrm{B}}=0 \quad \forall \mathrm{i}, \mathrm{t}$

(13) $\frac{\partial \mathrm{L}}{\partial \rho_{\mathrm{it}}}=\mathrm{K}_{\mathrm{it}}^{\mathrm{P}}-\theta \mathrm{K}_{\mathrm{it}-1}^{\mathrm{P}}+\mathrm{R}_{\mathrm{it}}-\mathrm{Pl}_{\mathrm{it}}=0 \quad \forall \mathrm{i}, \mathrm{t}$ 


\section{APENDICE III \\ LA DERIVACION DEL PRECIO DE RESERVA PARA LOS VENDEDORES \\ EN EL MERCADO SPOT}

\section{Definiciones}

Los precios de producción y del mercadospot son variables aleatorias con distribuciones conocidos. Se les puede representar de la siguiente manera:

$$
\begin{array}{lll}
\tilde{\mathrm{P}}_{\mathrm{t}}^{\mathrm{I}}=\overline{\mathrm{P}}_{\mathrm{t}}^{\mathrm{I}}+\mathrm{u}_{\mathrm{It}} & \mathrm{u}_{\mathrm{It}} \sim \mathrm{N}\left(0, \sigma_{\mathrm{I}}^{2}\right) & \mathrm{E}\left(\mathrm{u}_{\mathrm{It}} \mathrm{u}_{\mathrm{Wt}}\right)=0 \\
\tilde{\mathrm{P}}_{\mathrm{t}}^{\mathrm{W}}=\overline{\mathrm{P}}_{\mathrm{t}}^{\mathrm{W}}+\mathrm{u}_{\mathrm{Wt}} & \mathrm{u}_{\mathrm{Wt}} \sim \mathrm{N}\left(0, \sigma_{\mathrm{W}}^{2}\right)
\end{array}
$$

La varianza total en ingreso es igual a la varianza en el valor total de la producción regada más la varianza en el valor de las transacciones en el mercado spot. Se representa esta varianza como:

$$
\sigma_{\pi}^{2}=\sigma_{\mathrm{I}}^{2} \mathrm{f}(\bullet)^{2}+\sigma_{\mathrm{W}}^{2}\left(\mathrm{~W}_{\mathrm{it}}^{\mathrm{B}}\right)^{2}+\sigma_{\mathrm{W}}^{2}\left(\mathrm{~W}_{\mathrm{it}}^{\mathrm{S}}\right)^{2}
$$

\section{Derivación}

Se supone que $\mathrm{W}_{\mathrm{it}}^{\mathrm{S}}>0$ e se iguala a la ecuación (1) con la ecuación (3) del Apéndice II.

$$
\beta_{\mathrm{t}} \mathrm{E}\left[\mathrm{V}_{\mathrm{it}}^{\prime}\left(\tilde{\pi}_{\mathrm{it}}\right) \tilde{\mathrm{P}}_{\mathrm{t}}^{\mathrm{W}}\right]=\beta_{\mathrm{t}} \mathrm{E}\left\{\mathrm{V}_{\mathrm{it}}^{\prime}\left(\tilde{\pi}_{\mathrm{it}}\right)\left[\tilde{\mathrm{P}}_{\mathrm{t}}^{\mathrm{I}} \frac{\partial \mathrm{f}(\bullet)}{\partial \mathrm{e}_{\mathrm{i}} \mathrm{W}_{\mathrm{it}}^{\mathrm{I}}}-\frac{\partial \mathrm{c}(\bullet)}{\partial \mathrm{e}_{\mathrm{i}} \mathrm{W}_{\mathrm{it}}^{\mathrm{I}}}\right] \mathrm{e}_{\mathrm{i}}\right\}
$$

Combina los términos

$$
\mathrm{EV}_{\mathrm{it}}^{\prime}\left(\tilde{\pi}_{\mathrm{it}}\right)\left[\tilde{\mathrm{P}}_{\mathrm{t}}^{\mathrm{W}}-\left[\tilde{\mathrm{P}}_{\mathrm{t}}^{\mathrm{I}} \frac{\partial \mathrm{f}(\bullet)}{\partial \mathrm{e}_{\mathrm{i}} \mathrm{W}_{\mathrm{it}}^{\mathrm{I}}}-\frac{\partial \mathrm{c}(\bullet)}{\partial \mathrm{e}_{\mathrm{i}} \mathrm{W}_{\mathrm{it}}^{\mathrm{I}}}\right] \mathrm{e}_{\mathrm{i}}\right]=0
$$

Se aproxima la utilidad de ingreso estocástico por una expansión de Taylor Series alrededor del ingreso medio, donde $\tilde{\pi}_{\mathrm{it}}-\bar{\pi}_{\mathrm{it}}=\mathrm{u}_{\mathrm{Wt}} \mathrm{W}_{\mathrm{it}}^{\mathrm{S}}-\mathrm{u}_{\mathrm{Wt}} \mathrm{W}_{\mathrm{it}}^{\mathrm{B}}+\mathrm{u}_{\mathrm{It}} \mathrm{f}(\bullet)$

$$
\begin{aligned}
& \mathrm{E}\left[\mathrm{V}_{\mathrm{it}}^{\prime}\left(\overline{\mathrm{p}}_{\mathrm{it}}\right)\left\{\left(\overline{\mathrm{P}}_{\mathrm{t}}^{\mathrm{W}}+\mathrm{u}_{\mathrm{Wt}}\right)-\left[\left(\overline{\mathrm{P}}_{\mathrm{t}}^{\mathrm{I}}+\mathrm{u}_{\mathrm{It}}\right) \frac{\partial \mathrm{f}(\cdot)}{\partial \mathrm{e}_{\mathrm{i}} \mathrm{W}_{\mathrm{it}}^{\mathrm{I}}}-\frac{\partial \mathrm{c}(\cdot)}{\partial \mathrm{e}_{\mathrm{i}} \mathrm{W}_{\mathrm{it}}^{\mathrm{I}}}\right] \mathrm{e}_{\mathrm{i}}\right\}\right] \\
& +\mathrm{E}\left[\mathrm{V}_{\mathrm{it}}^{\prime \prime}\left(\overline{\mathrm{p}}_{\mathrm{it}}\right)\left[\left(\mathrm{u}_{\mathrm{Wt}} \mathrm{W}_{\mathrm{it}}^{\mathrm{S}}-\mathrm{u}_{\mathrm{Wt}} \mathrm{W}_{\mathrm{it}}^{\mathrm{B}}+\mathrm{uf}(\cdot)\right)\left\{\left(\overrightarrow{\mathrm{P}}_{\mathrm{t}}^{\mathrm{W}}+\mathrm{u}_{\mathrm{Wt}}\right)-\left[\left(\overline{\mathrm{P}}_{\mathrm{t}}^{\mathrm{I}}+\mathrm{u}_{\mathrm{It}}\right) \frac{\partial \mathrm{f}(\cdot)}{\partial \mathrm{e}_{\mathrm{i}} \mathrm{W}_{\mathrm{it}}^{\mathrm{I}}}-\frac{\partial \mathrm{c}(\cdot)}{\partial \mathrm{e}_{\mathrm{i}} \mathrm{W}_{\mathrm{it}}^{\mathrm{I}}}\right] \mathrm{e}_{\mathrm{i}}\right\}\right]\right]=0
\end{aligned}
$$


Se multiplica y se toman expectativas:

$$
\mathrm{V}_{\mathrm{it}}^{\prime}\left(\bar{\pi}_{\mathrm{it}}\right)\left[\overline{\mathrm{P}}_{\mathrm{t}}^{\mathrm{W}}-\left[\overline{\mathrm{P}}_{\mathrm{t}}^{\mathrm{I}} \frac{\partial \mathrm{f}(\bullet)}{\partial \mathrm{e}_{\mathrm{i}} \mathrm{W}_{\mathrm{it}}^{\mathrm{I}}} \frac{\partial \mathrm{c}(\bullet)}{\partial \mathrm{e}_{\mathrm{i}} \mathrm{W}_{\mathrm{it}}^{\mathrm{I}}}\right] \mathrm{e}_{\mathrm{i}}\right]+\mathrm{V}_{\mathrm{it}}^{\prime \prime}\left(\bar{\pi}_{\mathrm{it}}\right)\left[\sigma_{\mathrm{W}}^{2} \mathrm{~W}_{\mathrm{it}}^{\mathrm{S}}-\sigma_{\mathrm{W}}^{2} \mathrm{~W}_{\mathrm{it}}^{\mathrm{B}}-\sigma_{\mathrm{I}}^{2} \frac{\partial \mathrm{f}(\bullet)}{\partial \mathrm{e}_{\mathrm{i}} \mathrm{W}_{\mathrm{it}}^{\mathrm{I}}} \mathrm{e}_{\mathrm{i}} \mathrm{f}(\bullet)\right] \approx 0
$$

Si $\mathrm{W}_{\mathrm{it}}^{\mathrm{S}}>0$, entonces $\mathrm{W}_{\mathrm{it}}^{\mathrm{B}}$ debe ser igual a cero según las condiciones de primer orden. Por lo tanto, la ecuación anterior se reduce a:

$$
\mathrm{V}_{\mathrm{it}}^{\prime}\left(\bar{\pi}_{\mathrm{it}}\right)\left[\overline{\mathrm{P}}_{\mathrm{t}}^{\mathrm{W}}-\left[\overline{\mathrm{P}}_{\mathrm{t}}^{\mathrm{I}} \frac{\partial \mathrm{f}(\bullet)}{\partial \mathrm{e}_{\mathrm{i}} \mathrm{W}_{\mathrm{it}}^{\mathrm{I}}} \frac{\partial \mathrm{c}(\bullet)}{\partial \mathrm{e}_{\mathrm{i}} \mathrm{W}_{\mathrm{it}}^{\mathrm{I}}}\right] \mathrm{e}_{\mathrm{i}}\right]+\mathrm{V}_{\mathrm{it}}^{\prime \prime}\left(\bar{\pi}_{\mathrm{it}}\right)\left[\sigma_{\mathrm{W}}^{2} \mathrm{~W}_{\mathrm{it}}^{\mathrm{S}}-\sigma_{\mathrm{I}}^{2} \frac{\partial \mathrm{f}(\bullet)}{\partial \mathrm{e}_{\mathrm{i}} \mathrm{W}_{\mathrm{it}}^{\mathrm{I}}} \mathrm{e}_{\mathrm{i}} \mathrm{f}(\bullet)\right] \approx 0
$$

Se resuelve la ecuación por $\overline{\mathrm{P}}_{\mathrm{t}}^{\mathrm{W}}$, el precio esperado del mercado spot a tiempo t:

$$
\overline{\mathrm{P}}_{\mathrm{t}}^{\mathrm{W}} \approx\left[\overline{\mathrm{P}}_{\mathrm{t}}^{\mathrm{I}} \frac{\partial \mathrm{f}(\bullet)}{\partial \mathrm{e}_{\mathrm{i}} \mathrm{W}_{\mathrm{it}}^{\mathrm{I}}}-\frac{\partial \mathrm{c}(\bullet)}{\partial \mathrm{e}_{\mathrm{i}} \mathrm{W}_{\mathrm{it}}^{\mathrm{I}}}\right] \mathrm{e}_{\mathrm{i}}+\frac{\mathrm{V}_{\mathrm{it}}^{\prime \prime}\left(\bar{\pi}_{\mathrm{it}}\right)}{\mathrm{V}_{\mathrm{it}}^{\prime}\left(\bar{\pi}_{\mathrm{it}}\right)}\left[\sigma_{\mathrm{I}}^{2} \frac{\partial \mathrm{f}(\bullet)}{\partial \mathrm{e}_{\mathrm{i}} \mathrm{W}_{\mathrm{it}}^{\mathrm{I}}} \mathrm{e}_{\mathrm{i}} \mathrm{f}(\bullet)-\sigma_{\mathrm{W}}^{2} \mathrm{~W}_{\mathrm{it}}^{\mathrm{S}}\right]
$$

Un agricultor vende volúmenes de agua en el mercado spot cuando su precio de reserva, definido como el lado derecho de la ecuación anterior, es igual al precio esperado en el mercado spot. Se puede reescribir este precio de reserva como:

$$
\overline{\mathrm{P}}_{\mathrm{Rt}}^{\mathrm{SV}} \approx \mathrm{e}_{\mathrm{i}}\left[\overline{\mathrm{P}}_{\mathrm{t}}^{\mathrm{I}} \frac{\partial \mathrm{f}(\bullet)}{\partial \mathrm{e}_{\mathrm{i}} \mathrm{W}_{\mathrm{it}}^{\mathrm{I}}}-\frac{\partial \mathrm{c}(\bullet)}{\partial \mathrm{e}_{\mathrm{i}} \mathrm{W}_{\mathrm{it}}^{\mathrm{I}}}\right]-\frac{1}{2} \overline{\mathrm{R}}_{\mathrm{i}}\left[\frac{\partial \sigma_{\pi}^{2}}{\partial \mathrm{W}_{\mathrm{it}}^{\mathrm{I}}}-\frac{\partial \sigma_{\pi}^{2}}{\partial \mathrm{W}_{\mathrm{it}}^{\mathrm{S}}}\right]
$$

donde $\overline{\mathrm{R}}$ es el coeficiente “Arrow-Pratt” de aversión absoluta al riesgo, $\partial \sigma_{\pi}^{2} / \partial \mathrm{W}_{\mathrm{it}}^{\mathrm{I}}$ es el riesgo marginal asociado con un cambio en la cantidad de agua aplicada en riego, $\partial \sigma_{\pi}^{2} / \partial \mathrm{W}_{\mathrm{it}}^{\mathrm{S}}$ es el riesgo marginal asociado con vender volúmenes de agua en el mercado spot. 


\section{APENDICE IV}

LA DERIVACION DEL PRECIO DE RESERVA PARA LOS COMPRADORES

EN EL MERCADO SPOT

\section{Definiciones}

Los precios de producción y del mercadospot son variables aleatorias con distribuciones conocidos. Se les puede representar de la siguiente manera:

$$
\begin{array}{lll}
\tilde{\mathrm{P}}_{\mathrm{t}}^{\mathrm{I}}=\overline{\mathrm{P}}_{\mathrm{t}}^{\mathrm{I}}+\mathrm{u}_{\mathrm{It}} & \mathrm{u}_{\mathrm{It}} \sim \mathrm{N}\left(0, \mathrm{~s}_{\mathrm{I}}^{2}\right) & \mathrm{E}\left(\mathrm{u}_{\mathrm{It}} \mathrm{u}_{\mathrm{Wt}}\right)=0 \\
\tilde{\mathrm{P}}_{\mathrm{t}}^{\mathrm{W}}=\overline{\mathrm{P}}_{\mathrm{t}}^{\mathrm{W}}+\mathrm{u}_{\mathrm{Wt}} & \mathrm{u}_{\mathrm{Wt}} \sim \mathrm{N}\left(0, \mathrm{~s}_{\mathrm{W}}^{2}\right) &
\end{array}
$$

La varianza total en ingreso es igual a la varianza en el valor total de la producción regada más la varianza en el valor de las transacciones en el mercado spot. Se representa esta varianza como:

$$
\mathrm{s}_{\mathrm{p}}^{2}=\mathrm{s}_{\mathrm{I}}^{2} \mathrm{f}(\mathrm{x})^{2}+\mathrm{s} \underset{\mathrm{W}}{2}\left(\mathrm{~W}_{\mathrm{it}}^{\mathrm{B}}\right)^{2}+\mathrm{s}_{\mathrm{W}}^{2}\left(\mathrm{~W}_{\mathrm{it}}^{\mathrm{S}}\right)^{2}
$$

\section{Derivación}

Se supone que $\mathrm{W}_{\mathrm{it}}^{\mathrm{B}}>0 \mathrm{y}$ se iguala la ecuación (2) con la ecuación (3) del Apéndice II.

$$
\beta_{\mathrm{t}} \mathrm{E}\left[\mathrm{V}_{\mathrm{it}}^{\prime}\left(\tilde{\pi}_{\mathrm{it}}\right)\left(\tilde{\mathrm{P}}_{\mathrm{t}}^{\mathrm{W}}+\mathrm{tcw}\right)\right]=\beta_{\mathrm{t}} \mathrm{E}\left\{\mathrm{V}_{\mathrm{it}}^{\prime}\left(\tilde{\pi}_{\mathrm{it}}\right)\left[\tilde{\mathrm{P}}_{\mathrm{t}}^{\mathrm{I}} \frac{\partial \mathrm{f}(\cdot)}{\partial \mathrm{e}_{\mathrm{i}} \mathrm{W}_{\mathrm{it}}^{\mathrm{I}}}-\frac{\partial \mathrm{c}(\cdot)}{\partial \mathrm{e}_{\mathrm{i}} \mathrm{W}_{\mathrm{it}}^{\mathrm{I}}}\right] \mathrm{e}_{\mathrm{i}}\right\}
$$

Se combinan los términos:

$$
\mathrm{EV}_{\mathrm{it}}^{\prime}\left(\tilde{\pi}_{\mathrm{it}}\right)\left[\left(\tilde{\mathrm{P}}_{\mathrm{t}}^{\mathrm{W}}+\mathrm{tcw}\right)-\left[\tilde{\mathrm{P}}_{\mathrm{t}}^{\mathrm{I}} \frac{\partial \mathrm{f}(\cdot)}{\partial \mathrm{e}_{\mathrm{i}} \mathrm{W}_{\mathrm{it}}^{\mathrm{I}}} \frac{\partial \mathrm{c}(\cdot)}{\partial \mathrm{e}_{\mathrm{i}} \mathrm{W}_{\mathrm{it}}^{\mathrm{I}}}\right] \mathrm{e}_{\mathrm{i}}\right]=0
$$

Se aproxima la utilidad de ingreso estocástico por una expansión de Taylor Series alrededor del ingreso medio, donde $\tilde{p}_{i t}-\bar{p}_{i t}=u_{W t} W_{i t}^{S}-u_{w t} W_{i t}^{B}+u_{I t} f(\bullet)$

$$
\begin{aligned}
& \mathrm{E}\left[\mathrm{V}_{\mathrm{it}}^{\prime}\left(\overline{\mathrm{p}}_{\mathrm{it}}\right)\left\{\left(\overline{\mathrm{P}}_{\mathrm{t}}^{\mathrm{W}}+\mathrm{u}_{\mathrm{Wt}}\right)-\left[\left(\overline{\mathrm{P}}_{\mathrm{t}}^{\mathrm{I}}+\mathrm{u}_{\mathrm{It}}\right) \frac{\partial \mathrm{f}(\cdot)}{\partial \mathrm{e}_{\mathrm{i}} \mathrm{W}_{\mathrm{it}}^{\mathrm{I}}}-\frac{\partial \mathrm{c}(\cdot)}{\partial \mathrm{e}_{\mathrm{i}} \mathrm{W}_{\mathrm{it}}^{\mathrm{I}}}\right] \mathrm{e}_{\mathrm{i}}\right\}\right] \\
& +\mathrm{E}\left[\mathrm { V } _ { \mathrm { it } } ^ { \prime } ( \overline { \mathrm { p } } _ { \mathrm { it } } ) \left[\left(\mathrm{u}_{\mathrm{Wt}} \mathrm{W}_{\mathrm{it}}^{\mathrm{S}}-\mathrm{u}_{\mathrm{Wt}} \mathrm{W}_{\mathrm{it}}^{\mathrm{B}}+\mathrm{uf}(\cdot)\left\{\left(\overline{\mathrm{P}}_{\mathrm{t}} \mathrm{W}+\mathrm{u}_{\mathrm{Wt}}\right)-\left[\left(\overline{\mathrm{P}}_{\mathrm{t}}^{\mathrm{I}}+\mathrm{u}_{\mathrm{It}}\right) \frac{\partial \mathrm{f}(\cdot)}{\partial \mathrm{e}_{\mathrm{i}} \mathrm{W}_{\mathrm{it}}^{\mathrm{I}}}-\frac{\partial \mathrm{c}(\cdot)}{\partial \mathrm{e}_{\mathrm{i}} \mathrm{W}_{\mathrm{it}}^{\mathrm{I}}}\right] \mathrm{e}_{\mathrm{i}}\right\}\right]=0\right.\right.
\end{aligned}
$$


Se multiplica y se toman expectativas:

$\mathrm{V}_{\mathrm{it}}^{\prime}\left(\bar{\pi}_{\mathrm{it}}\right)\left[\overline{\mathrm{P}}_{\mathrm{t}}^{\mathrm{W}}+\mathrm{tcw}-\left[\overline{\mathrm{P}}_{\mathrm{t}}^{\mathrm{I}} \frac{\partial \mathrm{f}(\cdot)}{\partial \mathrm{e}_{\mathrm{i}} \mathrm{W}_{\mathrm{it}}^{\mathrm{I}}}-\frac{\partial \mathrm{c}(\cdot)}{\partial \mathrm{e}_{\mathrm{i}} \mathrm{W}_{\mathrm{it}}^{\mathrm{I}}}\right] \mathrm{e}_{\mathrm{i}}+\mathrm{V}_{\mathrm{it}}^{\prime \prime}\left(\bar{\pi}_{\mathrm{it}}\right)\left[\sigma_{\mathrm{W}}^{2} \mathrm{~W}_{\mathrm{it}}^{\mathrm{S}}-\sigma_{\mathrm{W}}^{2} \mathrm{~W}_{\mathrm{it}}^{\mathrm{B}}-\sigma_{\mathrm{I}}^{2} \frac{\partial \mathrm{f}(\cdot)}{\partial \mathrm{e}_{\mathrm{i}} \mathrm{W}_{\mathrm{it}}^{\mathrm{I}}} \mathrm{e}_{\mathrm{i}}-\mathrm{f}(\cdot)\right] \approx 0\right.$

Si $\mathrm{W}_{\mathrm{it}}^{\mathrm{B}}>0$, entonces $\mathrm{W}_{\mathrm{it}}^{\mathrm{S}}$ debe ser igual a cero según las condiciones de primer orden. Por lo tanto, la ecuación anterior se reduce a:

$$
\mathrm{V}_{\mathrm{it}}^{\prime}\left(\pi_{\mathrm{it}}\right)\left[\overline{\mathrm{P}}_{\mathrm{t}}^{\mathrm{W}}+\mathrm{tcw}-\left[\overline{\mathrm{P}}_{\mathrm{t}}^{\mathrm{I}}\left[\frac{\partial \mathrm{f}(\bullet)}{\partial \mathrm{e}_{\mathrm{i}} \mathrm{W}_{\mathrm{it}}^{\mathrm{I}}}-\frac{\partial \mathrm{c}(\bullet)}{\partial \mathrm{e}_{\mathrm{i}} \mathrm{W}_{\mathrm{it}}^{\mathrm{I}}}\right] \mathrm{e}_{\mathrm{i}}+\mathrm{V}_{\mathrm{it}}^{\prime}\left(\pi_{\mathrm{it}}\right)\left[\sigma_{\mathrm{W}}^{2} \mathrm{~W}_{\mathrm{it}}^{\mathrm{B}}-\sigma_{\mathrm{W}}^{2} \frac{\partial \mathrm{f}(\bullet)}{\partial \mathrm{e}_{\mathrm{i}} \mathrm{W}_{\mathrm{it}}^{\mathrm{I}}} \mathrm{e}_{\mathrm{i}} \mathrm{f}(\bullet)\right]\right] \approx 0\right.
$$

Se resuelve por $\overline{\mathrm{P}}_{\mathrm{t}}^{\mathrm{W}}$, el precio esperado del mercado spot a tiempo t:

$$
\overline{\mathrm{P}}_{\mathrm{t}}^{\mathrm{W}} \approx\left[\overline{\mathrm{P}}_{\mathrm{t}}^{\mathrm{I}} \frac{\partial \mathrm{f}(\bullet)}{\partial \mathrm{e}_{\mathrm{i}} \mathrm{W}_{\mathrm{it}}^{\mathrm{I}}}-\frac{\partial \mathrm{c}(\bullet)}{\partial \mathrm{e}_{\mathrm{i}} \mathrm{W}_{\mathrm{it}}^{\mathrm{I}}}\right] \mathrm{e}_{\mathrm{i}}+\frac{\mathrm{V}_{\mathrm{it}}^{\mathrm{\prime}}\left(\bar{\pi}_{\mathrm{it}}\right)}{\mathrm{V}_{\mathrm{it}}^{\prime}\left(\bar{\pi}_{\mathrm{it}}\right)}\left[\sigma_{\mathrm{I}}^{2} \frac{\partial \mathrm{f}(\bullet)}{\partial \mathrm{e}_{\mathrm{i}} \mathrm{W}_{\mathrm{it}}^{\mathrm{I}}} \mathrm{e}_{\mathrm{i}} \mathrm{f}(\bullet)+\sigma_{\mathrm{W}}^{2} \mathrm{~W}_{\mathrm{it}}^{\mathrm{B}}\right]-\mathrm{tcw}
$$

Un agricultor comprará volúmenes de agua en el mercado spot cuando su precio de reserva, definido como el lado derecho de la ecuación anterior, sea igual al precio esperado en el mercadospot, menos los costos de transacción. Se puede rescribir este precio de reserva como:

$$
\overline{\mathrm{P}}_{\mathrm{Rt}}^{\mathrm{SC}} \approx \mathrm{e}_{\mathrm{i}}\left[\overline{\mathrm{P}}_{\mathrm{t}}^{\mathrm{I}} \frac{\partial \mathrm{f}(\bullet)}{\partial \mathrm{e}_{\mathrm{i}} \mathrm{W}_{\mathrm{it}}^{\mathrm{I}}}-\frac{\partial \mathrm{c}(\bullet)}{\partial \mathrm{e}_{\mathrm{i}} \mathrm{W}_{\mathrm{it}}^{\mathrm{I}}}\right]-\frac{1}{2} \overline{\mathrm{R}}_{\mathrm{i}}\left[\frac{\partial \sigma_{\pi}^{2}}{\partial \mathrm{W}_{\mathrm{it}}^{\mathrm{I}}}+\frac{\partial \sigma_{\pi}^{2}}{\partial \mathrm{W}_{\mathrm{it}}^{\mathrm{B}}}\right]-\mathrm{tcw}
$$

donde $\bar{R}$ es el coeficiente "Arrow-Pratt” de aversión absoluta al riesgo, $\partial \sigma_{\pi}^{2} / \partial \mathrm{W}_{\mathrm{it}}^{\mathrm{I}}$ es el riesgo marginal asociado con un cambio en la cantidad de agua aplicada en riego, y $\partial \sigma_{\pi}^{2} / \partial \mathrm{W}_{\mathrm{it}}^{\mathrm{B}}$ es el riesgo marginal asociado con comprar volúmenes de agua en el mercado spot. 


\section{APENDICE V \\ DERIVACION DEL PRECIO DE RESERVA PARA UN DERECHO \\ DE APROVECHAMIENTO DE AGUA}

\section{Definiciones}

Los precios de producción y del mercadospot son variables aleatorias con distribuciones conocidas. Se les puede representar de la siguiente manera:

$$
\begin{array}{lcl}
\tilde{\mathrm{P}}_{\mathrm{t}}^{\mathrm{I}}=\overline{\mathrm{P}}_{\mathrm{t}}^{\mathrm{I}}+\mathrm{u}_{\mathrm{It}} & \mathrm{u}_{\mathrm{It}} \sim \mathrm{N}\left(0, \sigma_{\mathrm{I}}^{2}\right) & \mathrm{E}\left(\mathrm{u}_{\mathrm{It}} \mathrm{u}_{\mathrm{Wt}}\right)=0 \\
\tilde{\mathrm{P}}_{\mathrm{t}}^{\mathrm{W}}=\overline{\mathrm{P}}_{\mathrm{t}}^{\mathrm{W}}+\mathrm{u}_{\mathrm{Wt}} & \mathrm{u}_{\mathrm{Wt}} \sim \mathrm{N}\left(0, \sigma_{\mathrm{W}}^{2}\right) &
\end{array}
$$

La varianza total en ingreso es igual a la varianza en el valor total de la producción regada más la varianza en el valor de las transacciones en el mercado spot. Se representa esta varianza como:

$$
\sigma_{\pi}^{2}=\sigma_{I}^{2} f(\cdot)^{2}+\sigma_{W}^{2}\left(W_{i t}^{B}\right)^{2}+\sigma_{W}^{2}\left(W_{i t}^{S}\right)^{2}
$$

\section{Derivación}

Se resuelve la ecuación (3) por $\gamma_{\text {it }}$ y se sustituye la solución en la ecuación (6) del Apéndice II:

$$
\beta_{\mathrm{t}} \mathrm{EV} V_{\mathrm{it}}^{\prime}\left(\tilde{\pi}_{\mathrm{it}}\right)\left[-\mathrm{c}_{\mathrm{t}}^{\mathrm{R}}\right]+\mathrm{E}\left(\tilde{\omega}_{\mathrm{t}}\right)\left[\beta_{\mathrm{E}} \mathrm{EV} V_{\mathrm{it}}^{\prime}\left(\tilde{\pi}_{\mathrm{it}}\right)\left(\tilde{\mathrm{P}}_{\mathrm{t}}^{\mathrm{I}} \frac{\partial \mathrm{f}(\cdot)}{\partial \mathrm{e}_{\mathrm{i}} \mathrm{W}_{\mathrm{it}}^{\mathrm{I}}}-\frac{\partial \mathrm{c}(\cdot)}{\partial \mathrm{e}_{\mathrm{i}} \mathrm{W}_{\mathrm{it}}^{\mathrm{I}}}\right) \mathrm{e}_{\mathrm{i}}\right]-\lambda_{\mathrm{it}}+\lambda_{\mathrm{it}+1}=0
$$

Se combina con los términos:

$$
\beta_{\mathrm{t}} \mathrm{EV}_{\mathrm{it}}^{\prime}\left(\tilde{\pi}_{\mathrm{it}}\right)\left[\left(\tilde{\mathrm{P}}_{\mathrm{t}}^{\mathrm{I}} \frac{\partial \mathrm{f}(\cdot)}{\partial \mathrm{e}_{\mathrm{i}} \mathrm{W}_{\mathrm{it}}^{\mathrm{I}}}-\frac{\partial \mathrm{c}(\cdot)}{\partial \mathrm{e}_{\mathrm{i}} \mathrm{W}_{\mathrm{it}}^{\mathrm{I}}}\right) \mathrm{e}_{\mathrm{i}} \mathrm{E}\left(\tilde{\omega}_{\mathrm{t}}\right)-\mathrm{c}_{\mathrm{t}}^{\mathrm{R}}\right]=\lambda_{\mathrm{it}}-\lambda_{\mathrm{it}+1}
$$

Se resuelve la ecuación anterior por $\lambda_{\text {it }}$ a través de la sustitución recursiva:

$$
\lambda_{\mathrm{it}}=\sum_{\mathrm{t}=1}^{\mathrm{T}} \beta_{\mathrm{t}} E V_{\mathrm{it}}^{\prime}\left(\tilde{\pi}_{\mathrm{it}}\right)\left[\left(\tilde{\mathrm{P}}_{\mathrm{t}}^{\mathrm{I}} \frac{\partial \mathrm{f}(\cdot)}{\partial \mathrm{e}_{\mathrm{i}} \mathrm{W}_{\mathrm{it}}^{\mathrm{I}}}-\frac{\partial \mathrm{c}(\cdot)}{\partial \mathrm{e}_{\mathrm{i}} \mathrm{W}_{\mathrm{it}}^{\mathrm{I}}}\right) \mathrm{e}_{\mathrm{i}} \mathrm{E}\left(\tilde{\omega}_{\mathrm{i}}\right)-\mathrm{c}_{\mathrm{t}}^{\mathrm{R}}\right]+\lambda_{\mathrm{iT}}
$$

Se recuerda que según las condiciones de primer orden, $\lambda_{\mathrm{iT}}=\beta_{\mathrm{T}+1} \mathrm{~V}_{\mathrm{i}}^{\prime}\left(\pi_{\mathrm{iT}+1}\right) \mathrm{P}_{\mathrm{T}+1}^{\mathrm{R}}$. Se sustituye esta expresión en la ecuación anterior, rindiendo: 


$$
\lambda_{i t}=\sum_{t=1}^{T} \beta_{t} E V_{i t}^{\prime}\left(\tilde{\pi}_{i t}\right)\left[\left(\tilde{P}_{t}^{I} \frac{\partial f(\cdot)}{\partial e_{i} W_{i t}^{I}}-\frac{\partial c(\cdot)}{\partial e_{i} W_{i t}^{I}}\right) e_{i} E\left(\tilde{\omega}_{t}\right)-c_{t}^{R}\right]+\beta_{T+1} V_{i T+1}^{\prime}\left(\pi_{i T+1}\right) P_{i T+1}^{R}
$$

Se supone que $R_{\text {it }}^{S}>0$ y se sustituye la ecuación anterior en la ecuación (4) del Apéndice II:

$\beta_{\mathrm{t}} \mathrm{E} V_{1}^{\prime}\left(\tilde{\pi}_{\mathrm{it}}\right) \mathrm{P}_{\mathrm{t}}^{\mathrm{R}}=\sum_{\mathrm{t}=1}^{\mathrm{T}} \beta_{\mathrm{t}} \mathrm{E} \mathrm{V}_{1}^{\prime}\left(\tilde{\pi}_{\mathrm{it}}\right)\left[\left(\tilde{\mathrm{P}}_{\mathrm{t}}^{\mathrm{I}} \frac{\partial \mathrm{f}(\cdot)}{\partial \mathrm{e}_{\mathrm{i}} \mathrm{W}_{\mathrm{it}}^{\mathrm{I}}}-\frac{\partial \mathrm{c}(\cdot)}{\partial \mathrm{e}_{\mathrm{i}} \mathrm{W}_{\mathrm{it}}^{\mathrm{I}}}\right) \mathrm{e}_{\mathrm{i}} \mathrm{E}\left(\tilde{\omega}_{\mathrm{t}}\right)-\mathrm{c}_{\mathrm{t}}^{\mathrm{R}}\right]+\beta_{\mathrm{T}+1} \mathrm{~V}_{\mathrm{i}}^{\prime}\left(\pi_{\mathrm{iT}+1}\right) \mathrm{P}_{\mathrm{T}+1}^{\mathrm{R}}$

Se toma una expansión de Taylor Series alrededor del ingreso medio, tomando en cuenta que $\tilde{\mathrm{P}}_{\mathrm{t}}^{\mathrm{I}}=\overline{\mathrm{P}}_{\mathrm{t}}^{\mathrm{I}}+\mathrm{u}_{\mathrm{It}}$.

$$
\begin{aligned}
& \beta_{\mathrm{t}} \mathrm{E}\left[\mathrm{V}_{\mathrm{it}}^{\prime}\left(\bar{\pi}_{\mathrm{it}}\right)+\mathrm{V}_{\mathrm{it}}^{\prime \prime}\left(\bar{\pi}_{\mathrm{it}}\right)\left[\mathrm{u}_{\mathrm{Wt}}\left(\mathrm{W}_{\mathrm{it}}^{\mathrm{S}}-\mathrm{W}_{\mathrm{it}}^{\mathrm{B}}\right)+\mathrm{u}_{1 \mathrm{t}} \mathrm{f}(\bullet)\right]\right] \mathrm{P}_{\mathrm{t}}^{\mathrm{R}} \\
& =\sum_{\mathrm{t}=1}^{\mathrm{T}} \beta_{\mathrm{t}}\left[\mathrm{EV}_{\mathrm{it}}^{\prime}\left(\bar{\pi}_{\mathrm{it}}\right)+\mathrm{EV}_{\mathrm{it}}^{\prime \prime}\left(\bar{\pi}_{\mathrm{it}}\right)\right]\left[\left[\left(\overline{\mathrm{P}}_{\mathrm{t}}^{\mathrm{I}}+\mathrm{u}_{1 \mathrm{t}}\right) \frac{\partial \mathrm{f}(\bullet)}{\partial \mathrm{e}_{\mathrm{i}} \mathrm{W}_{\mathrm{it}}^{\mathrm{I}}}-\frac{\partial \mathrm{c}(\bullet)}{\partial \mathrm{e}_{\mathrm{i}} \mathrm{W}_{\mathrm{it}}^{\mathrm{I}}}\right] \mathrm{e}_{\mathrm{i}} \mathrm{E}\left(\tilde{\omega}_{\mathrm{t}}\right)-\mathrm{c}_{\mathrm{t}}^{\mathrm{R}}\right] \\
& +\mathrm{EV}_{\mathrm{it}}^{\prime \prime}\left(\bar{\pi}_{\mathrm{it}}\right)\left[\mathrm{u}_{\mathrm{Wt}}\left(\mathrm{W}_{\mathrm{it}}^{\mathrm{S}}-\mathrm{W}_{\mathrm{it}}^{\mathrm{B}}\right)+\mathrm{u}_{1 \mathrm{t}} \mathrm{f}(\bullet)\right]+\beta_{\mathrm{T}+1} \mathrm{~V}_{\mathrm{iT}+1}^{\prime}\left(\pi_{\mathrm{iT}+1}\right) \mathrm{P}_{\mathrm{T}+1}^{\mathrm{R}}
\end{aligned}
$$

Se multiplica y se toman expectativas:

$$
\begin{aligned}
\beta_{\mathrm{t}} \mathrm{V}_{\mathrm{it}}^{\prime}\left(\bar{\pi}_{\mathrm{it}}\right) \mathrm{P}_{\mathrm{t}}^{\mathrm{R}} & =\sum_{\mathrm{t}=1}^{\mathrm{T}} \beta_{\mathrm{t}} \mathrm{V}_{\mathrm{it}}^{\prime}\left(\bar{\pi}_{\mathrm{it}}\right)\left[\left(\overline{\mathrm{P}}_{\mathrm{t}}^{\mathrm{I}} \frac{\partial \mathrm{f}(\cdot)}{\partial \mathrm{e}_{\mathrm{i}} \mathrm{W}_{\mathrm{it}}^{\mathrm{I}}}-\frac{\partial \mathrm{c}(\cdot)}{\partial \mathrm{e}_{\mathrm{i}} \mathrm{W}_{\mathrm{it}}^{\mathrm{I}}}\right) \mathrm{e}_{\mathrm{i}} \overline{\boldsymbol{\omega}}_{\mathrm{t}}-\mathrm{c}_{\mathrm{t}}^{\mathrm{R}}\right] \\
& +\mathrm{V}_{\mathrm{it}}^{\prime \prime}\left(\bar{\pi}_{\mathrm{it}}\right)\left[\sigma_{\mathrm{I}}^{2} \frac{\partial \mathrm{f}(\cdot)}{\partial \mathrm{e}_{\mathrm{i}} \mathrm{W}_{\mathrm{it}}^{\mathrm{I}}} \mathrm{f}(\cdot) \mathrm{e}_{\mathrm{i}} \bar{\omega}_{\mathrm{t}}\right]+\beta_{\mathrm{T}+1} \mathrm{~V}_{\mathrm{i} \mathrm{T}+1}^{\prime}\left(\pi_{\mathrm{i} T+1}\right) \mathrm{P}^{\mathrm{R}}+1
\end{aligned}
$$

Se puede reescribir como:

$$
\beta_{\mathrm{t}} \mathrm{V}_{\mathrm{it}}^{\prime}\left(\bar{\pi}_{\mathrm{it}}\right) \mathrm{P}_{\mathrm{t}}^{\mathrm{R}}=\sum_{\mathrm{t}=1}^{\mathrm{T}} \beta_{\mathrm{t}} \mathrm{V}_{\mathrm{it}}^{\mathrm{t}}\left(\bar{\pi}_{\mathrm{it}}\right)\left[\begin{array}{l}
\left(\overline{\mathrm{P}}_{\mathrm{t}}^{\mathrm{I}} \frac{\partial \mathrm{f}(\bullet)}{\partial \mathrm{e}_{\mathrm{i}} \mathrm{W}_{\mathrm{it}}^{\mathrm{I}}}-\frac{\partial \mathrm{c}(\bullet)}{\partial \mathrm{e}_{\mathrm{i}} \mathrm{W}_{\mathrm{it}}^{\mathrm{I}}} \mathrm{e}_{\mathrm{i}} \bar{\omega}_{\mathrm{t}}-\mathrm{c}_{\mathrm{t}}^{\mathrm{R}}\right. \\
+\frac{\mathrm{V}_{\mathrm{it}}^{\prime \prime}\left(\bar{\pi}_{\mathrm{it}}\right)}{\mathrm{V}_{\mathrm{it}}^{\prime}\left(\bar{\pi}_{\mathrm{it}}\right)} \sigma_{\mathrm{I}}^{2} \frac{\partial \mathrm{f}(\bullet)}{\partial \mathrm{e}_{\mathrm{i}} \mathrm{W}_{\mathrm{it}}^{\mathrm{I}}} \mathrm{f}(\bullet) \mathrm{e}_{\mathrm{i}} \bar{\omega}_{\mathrm{t}}
\end{array}\right]+\beta_{\mathrm{T}+1} \mathrm{~V}_{\mathrm{iT}+1}^{\prime}\left(\pi_{\mathrm{iT}+1}\right) \mathrm{P}_{\mathrm{T}+1}^{\mathrm{R}}
$$

Se recuerda que $\sigma_{\mathrm{I}}^{2} \frac{\partial \mathrm{f}(\cdot)}{\partial \mathrm{e}_{\mathrm{i}} \mathrm{W}_{\mathrm{it}}^{\mathrm{I}}} \mathrm{f}() \mathrm{e}_{\mathrm{i}}=\frac{1}{2} \frac{\partial \sigma_{\pi}^{2}}{\partial \mathrm{W}_{\mathrm{it}}^{\mathrm{I}}}$ y $\frac{\mathrm{V}_{\mathrm{it}}^{\prime \prime}\left(\bar{\pi}_{\mathrm{it}}\right)}{\mathrm{V}_{\mathrm{it}}^{\prime}\left(\bar{\pi}_{\mathrm{it}}\right)}=-\overline{\mathrm{R}}_{\mathrm{i}}$, el negativo del coeficiente "Arrow-Pratt"de aversión al riesgo absoluta. Se sustituyen estas expresiones en la ecuación anterior, lo cual rinde: 


$$
\begin{aligned}
& \beta_{\mathrm{t}} \mathrm{V}_{\mathrm{it}}^{\prime}\left(\bar{\pi}_{\mathrm{it}}\right) \mathrm{P}_{\mathrm{t}}^{\mathrm{R}}=\sum_{\mathrm{t}=1}^{\mathrm{T}} \beta_{\mathrm{t}} \mathrm{V}_{\mathrm{it}}^{\prime}\left(\bar{\pi}_{\mathrm{it}}\right)\left[\left(\overline{\mathrm{P}}_{\mathrm{t}}^{\mathrm{I}} \frac{\partial \mathrm{f}(\bullet)}{\partial \mathrm{e}_{\mathrm{i}} \mathrm{W}_{\mathrm{it}}^{\mathrm{I}}}-\frac{\partial \mathrm{c}(\bullet)}{\partial \mathrm{e}_{\mathrm{i}} \mathrm{W}_{\mathrm{it}}^{\mathrm{I}}} \mathrm{e}_{\mathrm{i}} \bar{\omega}_{\mathrm{t}}-\mathrm{c}_{\mathrm{t}}^{\mathrm{R}}-\frac{1}{2} \overline{\mathrm{R}}_{\mathrm{i}} \frac{\partial \sigma_{\pi}^{2}}{\partial \mathrm{W}_{\mathrm{it}}^{\mathrm{I}}} \overline{\omega_{\mathrm{t}}}\right]\right. \\
& +\beta_{\mathrm{T}+1} \mathrm{~V}_{\mathrm{iT}+1}^{\prime}\left(\pi_{\mathrm{iT}+1}\right) \mathrm{P}_{\mathrm{T}+1}^{\mathrm{R}}
\end{aligned}
$$

Los precios de reserva de los derechos de aprovechamiento de agua son una función de las expectativas del agricultor de sus beneficios a futuro de agua de riego. En el tiempo t, estos beneficios a futuro son un flujo medio constante a través del tiempo. Por lo tanto, se puede reescribir la ecuación anterior como:

$$
\begin{aligned}
\frac{1}{(1+\mathrm{r})^{\mathrm{t}}} \mathrm{V}_{\mathrm{it}}^{\prime}\left(\bar{\pi}_{\mathrm{it}}\right) \mathrm{P}_{\mathrm{t}}^{\mathrm{R}} & =\left[\frac{1}{\mathrm{r}}-\frac{1}{\mathrm{r}(1+\mathrm{r})^{\mathrm{T}}}\right] \mathrm{V}_{\mathrm{it}}^{\prime}\left(\bar{\pi}_{\mathrm{it}}\right)\left[\left\{\left(\overline{\mathrm{P}}_{\mathrm{t}}^{\mathrm{I}} \frac{\partial \mathrm{f}(\bullet)}{\partial \mathrm{e}_{\mathrm{i}} \mathrm{W}_{\mathrm{it}}^{\mathrm{I}}}-\frac{\partial \mathrm{c}(\bullet)}{\partial \mathrm{e}_{\mathrm{i}} \mathrm{W}_{\mathrm{it}}^{\mathrm{I}}} \mathrm{e}_{\mathrm{i}} \overline{\omega_{\mathrm{t}}}-\mathrm{c}_{\mathrm{t}}^{\mathrm{R}}\right\}-\frac{1}{2} \overline{\mathrm{R}}_{\mathrm{i}} \frac{\partial \sigma_{\pi}^{2}}{\partial \mathrm{W}_{\mathrm{it}}^{\mathrm{I}}} \bar{\omega}_{\mathrm{t}}\right]\right. \\
& +\frac{1}{(1+\mathrm{r})^{\mathrm{T}+1}} \mathrm{~V}_{\mathrm{it}}^{\prime}\left(\bar{\pi}_{\mathrm{iT}+1}\right) \mathrm{P}_{\mathrm{T}+1}^{\mathrm{R}}
\end{aligned}
$$

Se resuelve por $\mathrm{P}_{\mathrm{t}}^{\mathrm{R}}$, rindiendo:

$$
\begin{aligned}
\mathrm{P}_{\mathrm{t}}^{\mathrm{R}} \approx & \frac{1}{(1+\mathrm{r})^{\mathrm{t}}}\left[\frac{1}{\mathrm{r}}-\frac{1}{\mathrm{r}(1+\mathrm{r})^{\mathrm{T}}}\right]\left[\left(\overline{\mathrm{P}}_{\mathrm{t}}^{\mathrm{I}} \frac{\partial \mathrm{f}(\bullet)}{\partial \mathrm{e}_{\mathrm{i}} \mathrm{W}_{\mathrm{it}}^{\mathrm{I}}}-\frac{\partial \mathrm{c}(\bullet)}{\partial \mathrm{e}_{\mathrm{i}} \mathrm{W}_{\mathrm{it}}^{\mathrm{I}}} \mathrm{e}_{\mathrm{i}} \bar{\omega}-\mathrm{c}_{\mathrm{t}}^{\mathrm{R}}-\frac{1}{2} \overline{\mathrm{R}}_{\mathrm{i}} \frac{\partial \sigma_{\pi}^{2}}{\partial \mathrm{W}_{\mathrm{it}}^{\mathrm{I}}} \bar{\omega}_{\mathrm{t}}\right]\right. \\
& +\frac{(1+\mathrm{r})^{\mathrm{t}}}{(1+\mathrm{r})^{\mathrm{T}+1}} \frac{\mathrm{V}_{\mathrm{iT}+1}^{\prime}\left(\pi_{\mathrm{iT}+1}\right) \mathrm{P}_{\mathrm{T}+1}^{\mathrm{R}}}{\mathrm{V}_{\mathrm{it}}^{\prime}\left(\bar{\pi}_{\mathrm{it}}\right)}
\end{aligned}
$$

Un agricultor venderá un derecho de aprovechamiento de agua cuando su precio de reserva, definido como el lado derecho de la ecuación anterior, sea igual al precio de derechos de aprovechamiento de agua en el mercado. Se reescribe esta expresión como:

$$
\begin{aligned}
& \overline{\mathrm{P}}_{\mathrm{R}}^{\mathrm{DAA}}=\frac{1}{\beta_{\mathrm{t}}} \mathrm{A}_{\mathrm{r}}^{\mathrm{T}}\left[\left\{\left(\overline{\mathrm{P}}_{\mathrm{t}}^{\mathrm{I}} \frac{\partial \mathrm{f}(\cdot)}{\partial \mathrm{e}_{\mathrm{i}} \mathrm{W}_{\mathrm{it}}^{\mathrm{I}}}-\frac{\partial \mathrm{c}(\cdot)}{\partial \mathrm{e}_{\mathrm{i}} \mathrm{W}_{\mathrm{it}}^{\mathrm{I}}}\right) \mathrm{e}_{\mathrm{i}} \bar{\omega}_{\mathrm{t}}-\mathrm{c}_{\mathrm{t}}^{\mathrm{R}}\right\}-\frac{1}{2} \overline{\mathrm{R}}_{\mathrm{i}} \frac{\partial \sigma_{\pi}^{2}}{\partial \mathrm{W}_{\mathrm{it}}^{\mathrm{I}}} \bar{\omega}_{\mathrm{t}}\right]+\beta_{\mathrm{T}} \frac{\mathrm{V}_{\mathrm{iT}+1}^{\prime}\left(\pi_{\mathrm{iT}+1}\right) \mathrm{P}_{\mathrm{T}}^{\mathrm{R}}}{\mathrm{V}_{\mathrm{iT}}^{\prime}\left(\bar{\pi}_{\mathrm{it}}\right)} \\
& \text { donde } \mathrm{A}_{\mathrm{t}}^{\mathrm{r}}=\left[\frac{1}{\mathrm{r}}-\frac{1}{(1+\mathrm{r})^{\mathrm{T}}}\right], \frac{1}{\beta_{\mathrm{t}}}=(1+\mathrm{r})^{\mathrm{t}}, \mathrm{y} \beta_{\mathrm{T}}=\frac{1}{(1+\mathrm{r})^{\mathrm{T}}}
\end{aligned}
$$

Para los compradores en el mercado de derechos de aprovechamiento de agua, el precio de reserva es igual al precio del mercado menos los costos de transacción, tcr. 


\section{APENDICE VI}

\section{LISTADO DE DEFINICIONES DE SIMBOLOS}

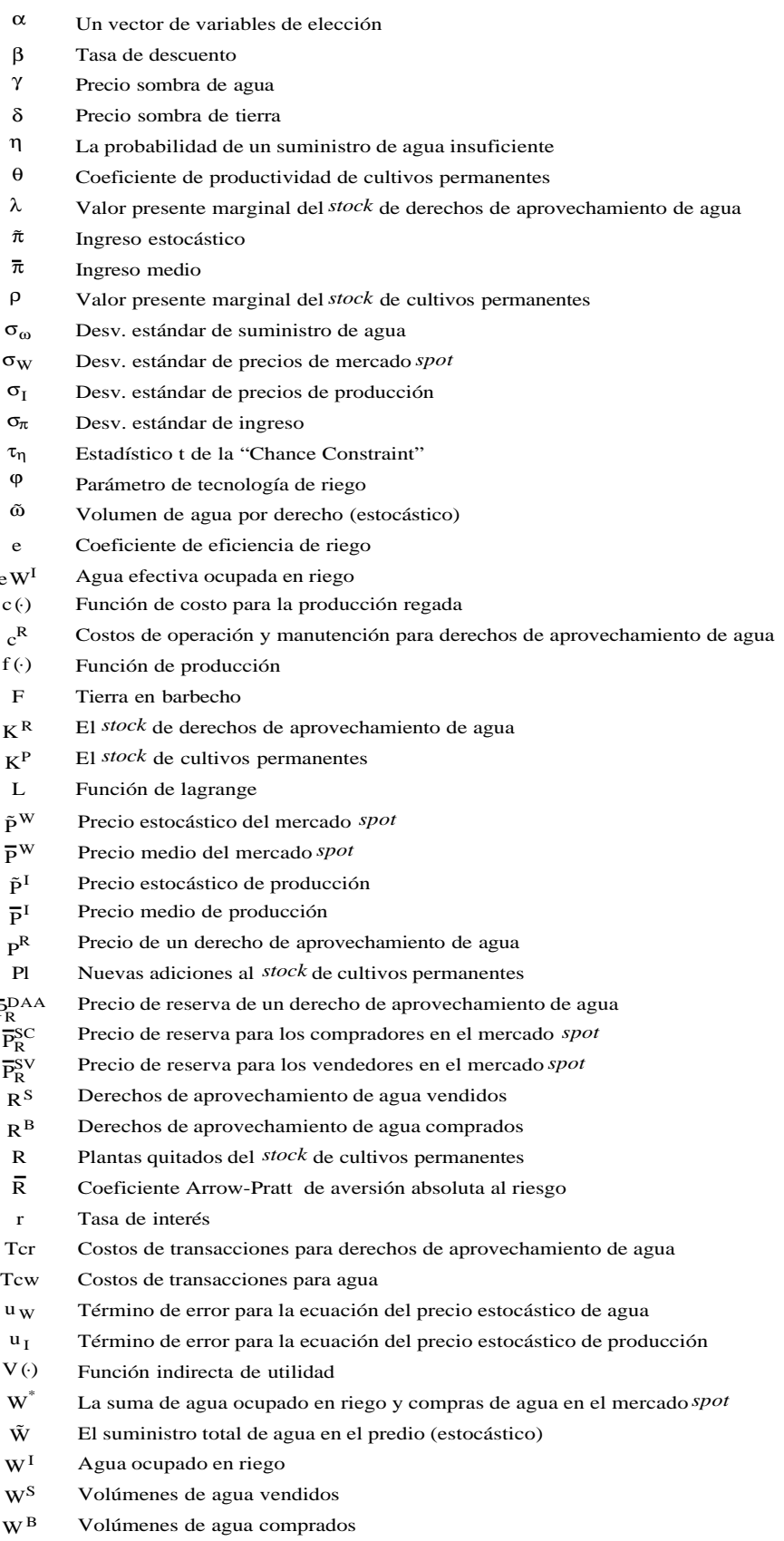

